\title{
GALACTIC MAGNETISM: Recent Developments and Perspectives
}

\author{
Rainer Beck
}

Max Planck Institute for Radioastronomy, Auf dem Hügel 69, D-53121 Bonn, Germany

Axel Brandenburg ${ }^{1}$

Nordita, Blegdamsvej 17, DK-2100 Copenhagen Ø, Denmark

\section{David Moss}

Mathematics Department, The University, Manchester M13 9PL, United Kingdom

Anvar Shukurov

Computing Center, Moscow University, 119899 Moscow, Russia

\section{Dmitry Sokoloff}

Department of Physics, Moscow University, 119899 Moscow, Russia

KEY WORDS: radio syncrotron emission, radio polarization, spiral arms, galactic halos, dynamos

\begin{abstract}
We discuss current observational and theoretical knowledge of magnetic fields, especially the large-scale structure in the disks and halos of spiral galaxies. Among other topics, we consider the enhancement of global magnetic fields in the interarm regions, magnetic spiral arms, and representations as superpositions of azimuthal modes, emphasizing a number of unresolved questions. It is argued that a turbulent hydromagnetic dynamo of some kind and an inverse cascade

${ }^{1}$ Now at Department of Mathematics and Statistics, University of Newcastle upon Tyne, NE1
\end{abstract} 7RU, United Kingdom. 
of magnetic energy gives the most plausible explanation for the regular galactic magnetic fields. Primordial theory is found to be unsatisfactory, and fields of cosmological origin may not even be able to provide a seed field for a dynamo. Although dynamo theory has its own problems, the general form of the dynamo equations appears quite robust. Finally, detailed models of magnetic field generation in galaxies, allowing for factors such as spiral structure, starbursts, galactic winds, and fountains, are discussed and confronted with observations.

\section{INTRODUCTION}

The magnetic field of the Milky Way has been investigated for about 40 years, and those of external spiral galaxies for about 20 years. It now seems clear that spiral galaxies generally possess large-scale magnetic fields whose evolution and, possibly, their origins are controlled by induction effects in the partially ionized interstellar gas. Turbulent motions with scales below about $100 \mathrm{pc}$ are present in this gas, and so the observed ubiquity of the large-scale galactic magnetic fields, coherent over scales of at least $1 \mathrm{kpc}$, requires special explanation. In fact, the theory of the galactic magnetic fields discussed in this review (known as mean-field magnetohydrodynamics) represents one of the earliest examples of synergetic theories describing how order can arise from chaos.

Our main emphasis is on magnetic fields whose scales exceed that of the interstellar turbulence. These are the fields-known as the mean, average, large-scale, global, or regular magnetic fields - that produce polarized radio emission in nearby spiral galaxies when observed at resolutions of $0.1-3 \mathrm{kpc}$. We also stress unresolved problems concerning the random (turbulent) magnetic fields in the interstellar medium (ISM), but we do not extend this discussion to the fields present in elliptical galaxies. Neither do we discuss phenomena connected with the central regions of the Milky Way.

The regular magnetic fields in the disks of spiral galaxies are usually considered to be the result of large-scale dynamo action, involving a collective inductive effect of turbulence (the $\alpha$-effect) and differential rotation. Even though alternatives to dynamo theory have been proposed, we believe that something resembling an $\alpha \Omega$-dynamo is the dominant mechanism, possibly sometimes modified by other hydromagnetic effects such as induction by streaming motions associated with spiral arms, other noncircular motions, and galactic fountains. The dynamo is the key ingredient of the theory: Other mechanisms by themselves are unable to maintain the observed large-scale galactic magnetic fields over galactic lifetimes.

The main rival of the dynamo theory is the primordial field theory. In this theory, one assumes that the observed magnetic patterns arise directly from a 
pregalactic magnetic field, distorted by the galactic differential rotation. We discuss why we believe that this theory, in spite of its appealing simplicity, cannot by itself give a detailed explanation of the range of field structures observed in spiral galaxies. A great conceptual advantage of the dynamo theory is that it can provide a universal explanation for the varied field configurations observed in spiral galaxies: axisymmetric and bisymmetric in azimuth; odd, even, and mixed parity vertically; etc. Of course, a primordial field may influence subsequent dynamo action, or it may be amplified by a dynamo.

The dynamo theory has its own difficulties. The linear version, which is valid when the magnetic field is too weak to significantly affect the velocity field, is relatively well developed and agrees favorably with observations wherever such a comparison is meaningful. However, the nonlinear saturation of the dynamo is not well understood and the conventional ideas were recently strongly criticized. They certainly need substantial improvement (Section 4). We argue, however, that the mathematical form of the mean-field dynamo equations is rather generic and robust, so that the available results are expected to be at least qualitatively correct, even though the details and the physical meaning of the coefficients of the dynamo equations may need to be revised.

The topics of this article have recently been reviewed by Wielebinski \& Krause (1993) and Kronberg (1994). We have attempted to avoid unnecessary repetition of their material.

\section{INTERPRETATION OF RADIO OBSERVATIONS}

Interstellar magnetic fields can be observed indirectly at optical and radio wavelengths. Heiles (1976), Verschuur (1979), and Tinbergen (1996) provide extensive reviews of observational methods. In recent years, observations of the linearly polarized radio continuum emission have improved significantly; these provide the most extensive and reliable information about galactic magnetic fields. We thus concentrate on results based on radio continuum data. Zeeman splitting measurements are discussed by Heiles et al (1993). For optical and infrared polarization data, see Roberge \& Whittet (1996).

\subsection{Field Strength Estimates}

The strengths of the projections of the total $(\mathbf{B})$ and regular $(\overline{\mathbf{B}})$ magnetic fields onto the plane of the sky $\left(\mathbf{B}_{\perp}\right.$ and $\left.\overline{\mathbf{B}}_{\perp}\right)$ can be determined from the intensity of the total and linearly polarized synchrotron emission (e.g. Rybicki \& Lightman 1979, p. 180). However, a relation between the energy densities of relativistic electrons, $\epsilon_{\mathrm{e}}$, and the total magnetic field, $\epsilon_{B}$, has to be assumed. Direct measurements of cosmic rays are possible only near the Earth. The local cosmic-ray 
energy density $\epsilon_{\mathrm{CR}}$ is comparable to $\epsilon_{B}$, and $K=\epsilon_{\mathrm{CR}} / \epsilon_{\mathrm{e}} \simeq 100$ locally, but is possibly lower in other galaxies (Pohl 1993).

It is plausible to assume $\epsilon_{\mathrm{CR}}=a \epsilon_{B}$, where $a$ depends on the detailed model: pressure equilibrium, minimum total energy, or energy density equipartition. Although the validity of these assumptions may be questioned (Longair 1994, Urbanik et al 1994, Heiles 1996), they generally provide reasonable estimates.

Gamma-ray observations have been used to obtain indirect data about the distribution of cosmic-ray electrons in the Galaxy (Bloemen et al 1986) and in the Magellanic Clouds (Chi \& Wolfendale 1993). Comparing radio and $\gamma$-ray data for the Magellanic Clouds, Chi \& Wolfendale claimed that energy equipartition is not valid (see, however, Pohl 1993). Their arguments would not apply if $\gamma$ and radio emissions originate from different regions.

The standard minimum-energy formulae generally use a fixed integration interval in frequency to determine the total energy density of cosmic-ray electrons. This procedure makes it difficult to compare minimum-energy field strengths between galaxies because a fixed frequency interval corresponds to different electron energy intervals, depending on the field strength itself. When a fixed integration interval in electron energy is used, the minimum-energy and energy equipartition estimates give similar values for $\left\langle B^{2} B_{\perp}^{1+\alpha_{\mathrm{s}}}\right\rangle \simeq\left\langle B_{\perp}^{3+\alpha_{\mathrm{s}}}\right\rangle$, where $\alpha_{\mathrm{s}}$ is the synchrotron spectral index (typically $\simeq 0.9$ ). The resulting estimate $\left\langle B_{\perp}^{3+\alpha_{\mathrm{s}}}\right\rangle^{1 /\left(3+\alpha_{\mathrm{s}}\right)}$ is larger than the mean field $\left\langle B_{\perp}\right\rangle$ if the field strength varies along the path length, since $\left\langle B_{\perp}\right\rangle^{3+\alpha_{\mathrm{s}}} \leq\left\langle B_{\perp}^{3+\alpha_{\mathrm{s}}}\right\rangle$. (Here and elsewhere we denote the magnitude of a vector by $B=|\mathbf{B}|$.)

If the field is concentrated in filaments with a volume filling factor $f$, the equipartition estimate is smaller than the field strength in the filaments by a factor $f^{1 /\left(3+\alpha_{s}\right)}$. The derived field strength depends on the power $\left(3+\alpha_{\mathrm{s}}\right)^{-1} \simeq$ $1 / 4$ of any of the input values, so that even large uncertainties cause only a moderate error in field strength. For example, a probable uncertainty in $K$ of $50 \%$ gives an error in magnetic field strength of $\simeq 15 \%$, with the total uncertainty perhaps reaching $30 \%$.

An estimate of the regular field strength $\bar{B}_{\perp}$ can be obtained by using the observed degree of polarization $P$, from $P \simeq P_{0}\left(\bar{B}_{\perp} / B_{\perp}\right)^{2}$, where $P_{0} \simeq 75 \%$ (Burn 1966). Note that regular field strengths are always lower limits because of limited instrumental resolution.

\subsection{Large-Scale Field Patterns}

The plane of polarization of a linearly polarized radio wave rotates when the wave passes through a plasma with a regular magnetic field. The rotation angle $\Delta \psi$ increases with the integral of $n_{\mathrm{e}} B_{\|}$along the line of sight (where $n_{\mathrm{e}}$ is the thermal electron density and $B_{\|}$is the component of the total magnetic field 
along the line of sight) and with $\lambda^{2}$ (where $\lambda$ is the wavelength of observation). The quantity $\Delta \psi / \Delta \lambda^{2}$ is called the rotation measure, RM. The observed RM is sensitive to the regular magnetic field $\bar{B}_{\|}$because the random fields $b_{\|}$ mostly cancel. The sign of RM allows the two opposite directions of $\bar{B}_{\|}$to be distinguished. An accurate determination of RM requires observations at (at least) three wavelengths because the observed orientation of the polarization plane is ambiguous by a multiple of $\pm 180^{\circ}$ (see Ruzmaikin \& Sokoloff 1979). Unlike equipartition estimates, which are insensitive to the presence of field reversals within the volume observed by the telescope beam, the observed value of Faraday rotation will decrease with increasing number of reversals.

Although the filled apertures of single-dish telescopes are sensitive to all spatial structures above the resolution limit, synthesis instruments such as the VLA cannot provide interferometric data at short spacings. This shortcoming results in some blindness to extended emission. Missing large-scale structures in maps of Stokes parameters $Q$ and $U$ can systematically distort the polarization angles and hence the RM distribution, so that the inclusion of additional data from single-dish telescopes in all Stokes parameters is required. In Section 3.4 (Figure 3) we show the result of such a successful combination by using a maximum-entropy method.

A convenient general way to parameterize the global magnetic field (irrespective of its origin) is by Fourier decomposition in terms of the azimuthal angle $\phi$ measured in the plane of the galaxy, $\overline{\mathbf{B}}=\sum_{m} \overline{\mathbf{B}}_{m} \exp (i m \phi)$. In practice, observations are analyzed within rings (centered at the galaxy's center) whose width is chosen to be consistent with the resolution of the observations. The result is a set of Fourier coefficients of the large-scale magnetic field for each ring. Usually, a combination of $m=0$ and $m=1$ modes is enough to provide a statistically satisfactory fit to the data. This is a remarkable indication of the presence of genuine global magnetic structures in spiral galaxies.

All observed magnetic fields have significant radial and azimuthal components: The magnetic lines of the regular field are spirals (Section 8.3). We distinguish between spiral structures that can be considered as basically axisymmetric (ASS), and basically antisymmetric or bisymmetric (BSS), with respect to rotation by $180^{\circ}$. Note that higher azimuthal Fourier modes are expected to be superimposed on these dominant ones, but these should have relatively small amplitudes. Fields containing several Fourier components of significant amplitude have mixed spiral structure (MSS); this might be considered to be a combination of ASS and BSS.

A further classification of magnetic structures according to their symmetry with respect to the galaxy's midplane distinguishes symmetric $S$ (i.e. even parity or quadrupole) from antisymmetric (odd parity or dipole) modes A. 
Table 1 A two-dimensional classification of global magnetic structures listing the dominant azimuthal and vertical modes

\begin{tabular}{lccc}
\hline \hline \multirow{2}{*}{$\begin{array}{l}\text { Vertical } \\
\text { structure }\end{array}$} & \multicolumn{3}{c}{ Azimuthal structure } \\
\cline { 2 - 4 } & ASS & BSS & MSS \\
\hline Even & S0 & S1 & S0 + S1 \\
Odd & A0 & A1 & A0 + A1 \\
Mixed & M0 & M1 & M0 + M1
\end{tabular}

Mixed-parity distributions (M), in which the magnetic fields are neither even nor odd but are superpositions, are also possible. This notation is supplemented with a value of the azimuthal wave number $m$, e.g. S0 means a quadrupole axisymmetric field. The notation used in discussions of global magnetic structures in spiral galaxies in presented in Table 1.

An ASS (BSS) field produces a $2 \pi$-periodic ( $\pi$-periodic) distribution of RM along $\phi$ (Sofue et al 1986, Krause 1990, Wielebinski \& Krause 1993). For the $m=0$ mode, the phase of the variation of RM with $\phi$ is equal to the magnetic pitch angle, $p=\arctan \left(\bar{B}_{r} / \bar{B}_{\phi}\right)$. Using the observed azimuthal distribution of $\mathrm{RM}$ in a galaxy, the structure of the line-of-sight component of a large-scale magnetic field can be studied. This method is difficult to apply if the data suffer from Faraday depolarization, if the regular field is not parallel to the plane of the galaxy, if its pitch angle in the disk is not constant, or if the disk is surrounded by a halo with magnetic fields of comparable strengths.

A more direct method of analysis considers polarization angles $\psi$ without converting them into Faraday rotation measures (Ruzmaikin et al 1990; Sokoloff et al 1992; EM Berkhuijsen et al, in preparation). There are three main contributions to the observed polarization angle: $\psi=\psi_{0}+\mathrm{RM} \lambda^{2}+\mathrm{RM}_{\mathrm{fg}} \lambda^{2}$, where $\psi_{0}$ is determined by the transverse magnetic field in the galaxy, RM is associated with Faraday rotation by the line-of-sight magnetic field in the galaxy, and $\mathrm{RM}_{\mathrm{fg}}$ is the foreground rotation measure. Thus, a direct analysis of $\psi$ patterns at several wavelengths allows a self-consistent study of all three components of the regular magnetic field. Another advantage of this method is that complicated magnetic structures along the line of sight can be studied. Implementations of this method employ consistent statistical tests such as the $\chi^{2}$ and Fisher criteria, thereby allowing the reliability of the results to be assessed.

Note that Faraday rotation analysis yields an average value, $\left\langle n_{\mathrm{e}} B_{\|}\right\rangle$. Information on $\bar{B}_{\|}$can be extracted only if a reliable model for the distribution of $n_{\mathrm{e}}$ is available, which is often not the case. If, for example, the thermal gas has a low filling factor, any result concerning $\bar{B}_{\|}$may not be representative. 


\subsection{Small-Scale Field Structures}

Any unresolved field structures will lead to beam depolarization and thus to polarizations significantly below the theoretical limit of $P_{0} \simeq 75 \%$. This effect is independent of wavelength and can be used to estimate the spatial scale and strength of field irregularities using observations at short wavelengths, where Faraday effects are weak.

At longer wavelengths, varying field orientations along the line of sight give rise to dispersion in rotation measures (Faraday dispersion), which also leads to depolarization (Burn 1966). Faraday dispersion is expected to arise from small H II regions (of $\simeq 1 \mathrm{pc}$ in size) in the thin galactic disk (Ehle \& Beck 1993) as well as from larger scale fluctuations $(\simeq 10-100 \mathrm{pc})$ in the diffuse ionized medium of the thick disk (e.g. Krause 1993, Neininger et al 1993). This effect makes the Faraday rotation angle no longer proportional to $\lambda^{2}$ because the effective Faraday depth decreases with increasing $\lambda$. It was recently discovered that at wavelengths greater than or equal to $10 \mathrm{~cm}$, galaxies are generally not transparent to polarized radio waves (Sukumar \& Allen 1991, Beck 1991, Horellou et al 1992). Even at $\lambda \simeq 6 \mathrm{~cm}$ complete Faraday depolarization may occur in spiral arms or in the plane of edge-on galaxies.

To obtain full rotation measures, only observations in the Faraday-thin regime $(\lambda \leq 6 \mathrm{~cm})$ should be used (Vallée 1980, Beck 1993). Rotation measures between longer wavelengths are lower and are weighted to regions near to the observer. Variations in Faraday depth may also lead to a spatial variation of the observed RM, which complicates the interpretation of observations. On the other hand, Faraday depolarization allows the study of layers at different depths sampled at different wavelengths (EM Berkhuijsen et al, in preparation).

\subsection{Comparison with Optical Polarization Data}

Optical polarization observations have revealed spiral magnetic patterns in M51 (Scarrott et al 1987), NGC 1068 (Scarrott et al 1991), NGC 1808 (Scarrott et al 1993), and other galaxies (Scarrott et al 1990) (see also the review by Hough 1996). In the western half of M51, field orientations as derived from optical polarization disagree by up to about $60^{\circ}$ from the spiral pattern as derived at several wavelengths in the radio continuum (Beck et al 1987). Optical polarization is contaminated by highly polarized light due to scattering at large angles. Polarization observations at far-infrared or submillimeter wavelengths are free from scattering effects and reveal the magnetic field structure in Galactic dust clouds (Davidson et al 1995) and near the Galactic Center (Hildebrand et al 1990, Hildebrand \& Davidson 1994). 


\section{MAGNETIC FIELDS IN SPIRAL GALAXIES}

\subsection{Field Strengths}

Mean equipartition strengths of the total field $\left\langle B^{3+\alpha_{\mathrm{s}}}\right\rangle^{1 /\left(3+\alpha_{\mathrm{s}}\right)}$ (averaged over the volume of the visible radio disk) range from $\simeq 4 \mu \mathrm{G}$ in M33 (Buczilowski \& Beck 1991) to $\simeq 12 \mu \mathrm{G}$ in NGC 6946 and NGC 1566 (Ehle \& Beck 1993, Ehle et al 1996); they are proportional to surface brightness in the far-infrared range (Hummel et al 1988b) and to average gas density (S Niklas et al, in preparation). Hummel's (1986) sample of $88 \mathrm{Sbc}$ galaxies has a mean minimum-energy field of $\simeq 8 \mu \mathrm{G}$, using $K=100$. Using the same value of $K$ for the sample of 146 late-type galaxies by Fitt \& Alexander (1993), one obtains a mean total minimum-energy field strength of $10 \pm 4 \mu \mathrm{G}$. Extremal values found in normal galaxies can be up to $20 \mu \mathrm{G}$ in spiral arms, as in NGC 6946 (Beck 1991) and NGC 1566 (Ehle et al 1996). In the mildly active galaxy M82, Klein et al (1988) found a field strength $\simeq 50 \mu \mathrm{G}$.

The regular field strengths $\bar{B}_{\perp}$ as obtained from the intensity of polarized emission are typically a few $\mu \mathrm{G}$. Such values are roughly consistent with regular field strengths $\bar{B}_{\|}$as derived from Faraday rotation data, if we assume typical electron densities of a few $10^{-2} \mathrm{~cm}^{-3}$ (see e.g. Buczilowski \& Beck 1991). Because polarized intensity and rotation measure depend differently on the filling factor of the field, the fact that $\bar{B}_{\perp} \simeq \bar{B}_{\|}$implies that the filling factor is not very small. The ratio of regular to turbulent field strengths is typically $\simeq 0.5$ if observed with a spatial resolution of a few kpc (Buczilowski \& Beck 1991).

In NGC 2276 the regular field strength reaches $10 \mu \mathrm{G}$ (Hummel \& Beck 1995), probably due to its interaction with the ambient intracluster gas. The total field is also unusually strong in that galaxy. Galaxies in clusters generally contain stronger fields (Gavazzi et al 1991, Niklas et al 1995).

\subsection{M31: The Nearly Perfect Magnetic Torus}

Radio observations of M31 reveal a $\simeq 20 \mathrm{kpc}$-diameter torus with the regular magnetic field, as determined from a Faraday rotation model, aligned uniformly with respect to the circumference (Sofue \& Takano 1981, Beck 1982, Beck et al 1989, Ruzmaikin et al 1990). A superposition of helical field loops (as expected from dynamo theory) can explain the asymmetric distribution of polarized emission with respect to the minor axis (Donner \& Brandenburg 1990, Urbanik et al 1994). The mean equipartition field strength in the torus is $\simeq 7 \mu \mathrm{G}$ for the total field and $\simeq 4 \mu \mathrm{G}$ for the regular field.

M31 has a low star-formation rate globally and no grand-design spiral structure. Strong compression of magnetic fields by density waves seems to be absent. However, there are many small dust lanes and cloud complexes, which 
are traced by field lines. Comparison between the total radio intensity and the gas emission in $\mathrm{CO}$ and $\mathrm{HI}$ confirms a close coupling of the field to the gas clouds (Berkhuijsen et al 1993).

\subsection{Density-Wave Galaxies}

Radio polarization observations show that the regular magnetic fields follow approximately the optical spiral structure in M81 (Krause et al 1989b), M83 (Neininger et al 1991, Ehle 1995), NGC 1566 (Ehle et al 1996), and M51 (Neininger 1992; Neininger \& Horellou 1996; EM Berkhuijsen et al, in preparation), but that the streamlines of the rotation models of the gas do not follow the optical spiral structure. The streamlines have strongly varying pitch angles (e.g. Otmianowska-Mazur \& Chiba 1995) and are almost closed in the disk. However, some regions of M51 may be exceptional: Neininger (1992) has claimed that some field lines are carried along with streaming motions. The field lines in the central region of M83 are aligned with the bar.

Strong shocks should compress the magnetic field and lead to high degrees of polarization of 40-70\% in the radio continuum (Beck 1982) at the inner edges of the spiral arms (see Section 8.4). Only in M51 are the strongest aligned fields indeed found at the positions of the prominent dust lanes on the inner edges of the optical spiral arms (Figure 1). This is best visible along the eastern arm where the aligned field even follows the dust lane crossing the optical arm. However, some regular fields extend far into the interarm regions. Furthermore, the $10-30 \%$ polarization at $\lambda 6 \mathrm{~cm}$ is in contrast to the higher polarizations expected from shock alignment. Hence the radio data only tell us that the regular fields in M51 are somehow coupled to the cool gas as traced by dust lanes.

The aligned fields in M81 and NGC 1566 are strongest in interarm regions (Krause et al 1989b, Ehle et al 1996), whereas the total synchrotron intensity (tracing the total field) is highest in the optical spiral arms. Strongly aligned interarm fields have also been detected in the outer parts of M83, where the star formation rate is low (Allen \& Sukumar 1990). High-resolution observations of M81 (Schoofs 1992; see Figure 2) confirmed that the regular fields extend across almost the entire interarm region, but are somewhat stronger near the inner edge of the prominent western spiral arm, where some dust clouds are visible. We stress that the distribution of magnetic pitch angles exhibits a weaker arm-interarm variation than that of the regular magnetic field strengths. Soida et al (1996) showed that strength and pitch angle of the regular fields in NGC 4254 reveal much less arm-interarm variations than expected from density-wave compression in its two major arms. They also showed that regular fields even exist in regions of chaotic optical pattern. 


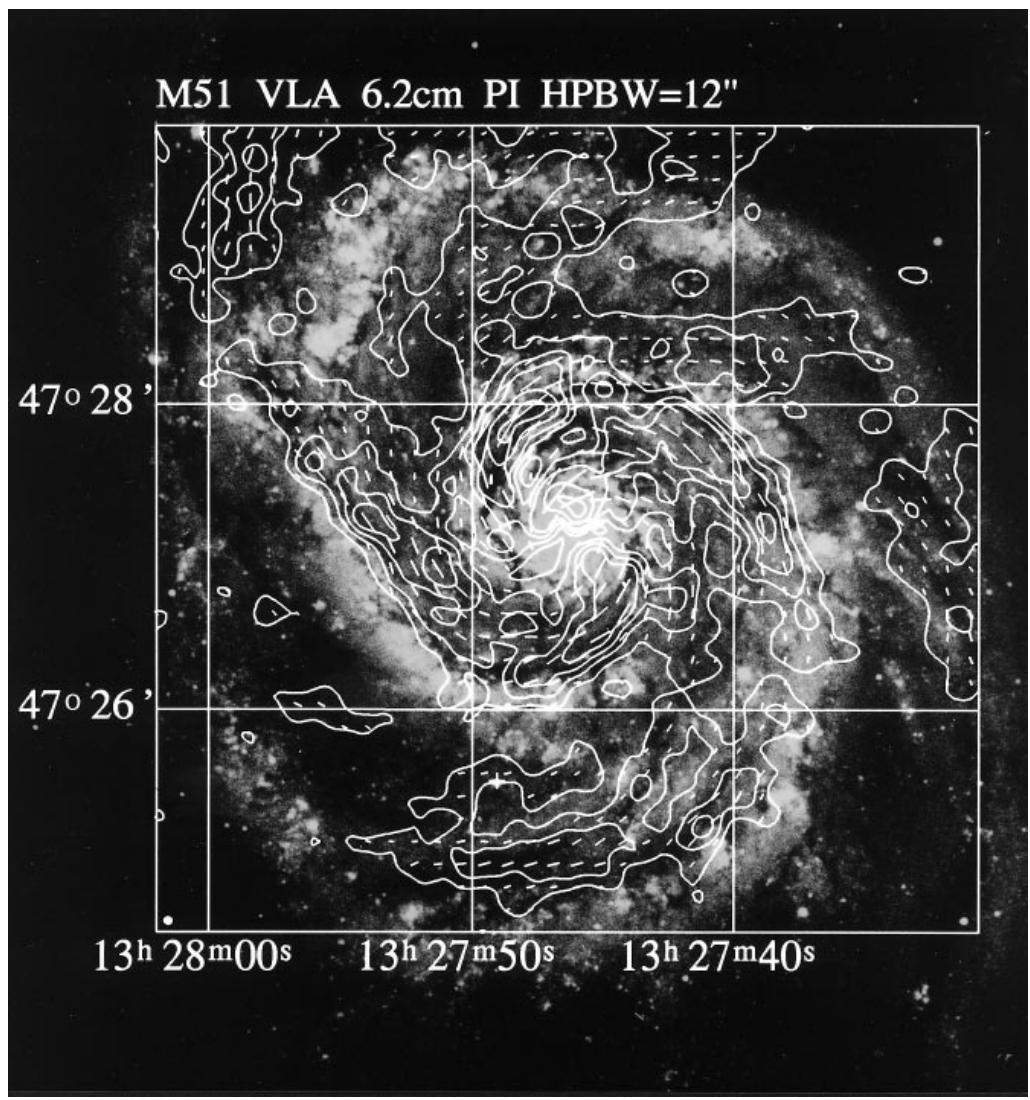

Figure 1 Polarized synchrotron intensity (contours) and magnetic field orientation of M51 (obtained by rotating the $E$-vectors by $90^{\circ}$ ), observed at $\lambda 6.2 \mathrm{~cm}$ with the VLA ( $12 \operatorname{arcsec}$ synthesized beam). (From Neininger \& Horellou 1996.)

\subsection{IC 342 and NGC 6946: Magnetic Spiral Arms}

These two galaxies exhibit high star-formation rates, but their spiral structure is less regular than that of M51. Long arms of polarized emission are present in IC 342 (Krause et al 1989a, Krause 1993).

Recent high-resolution observations of the similar galaxy NGC 6946 (Beck \& Hoernes 1996; see Figure 3) revealed a surprisingly symmetric distribution of polarized emission with two major spiral features, in the north and in the south, located between optical spiral arms and running perfectly parallel to the adjacent optical arms over at least $12 \mathrm{kpc}$. This regular two-armed structure is much more symmetric than the distribution of total field, gas, and stars, which 
all show a quite irregular, multiarmed pattern. Two further, weaker, magnetic spiral arms are visible between the two main ones (Figure 3).

The main magnetic spiral arms in NGC 6946 do not fill all of the interarm regions, unlike the polarized emission in M81, but are only about $0.5-1 \mathrm{kpc}$ wide. As they are also visible in total emission, both the regular and total magnetic fields are enhanced there. The strength of the (resolved) regular field varies between 3 and $13 \mu \mathrm{G}$ along the arm. The peak values of polarized intensity and degree of polarization occur in the northern magnetic arm of NGC 6946, in a region between the optical arms, where the density of warm

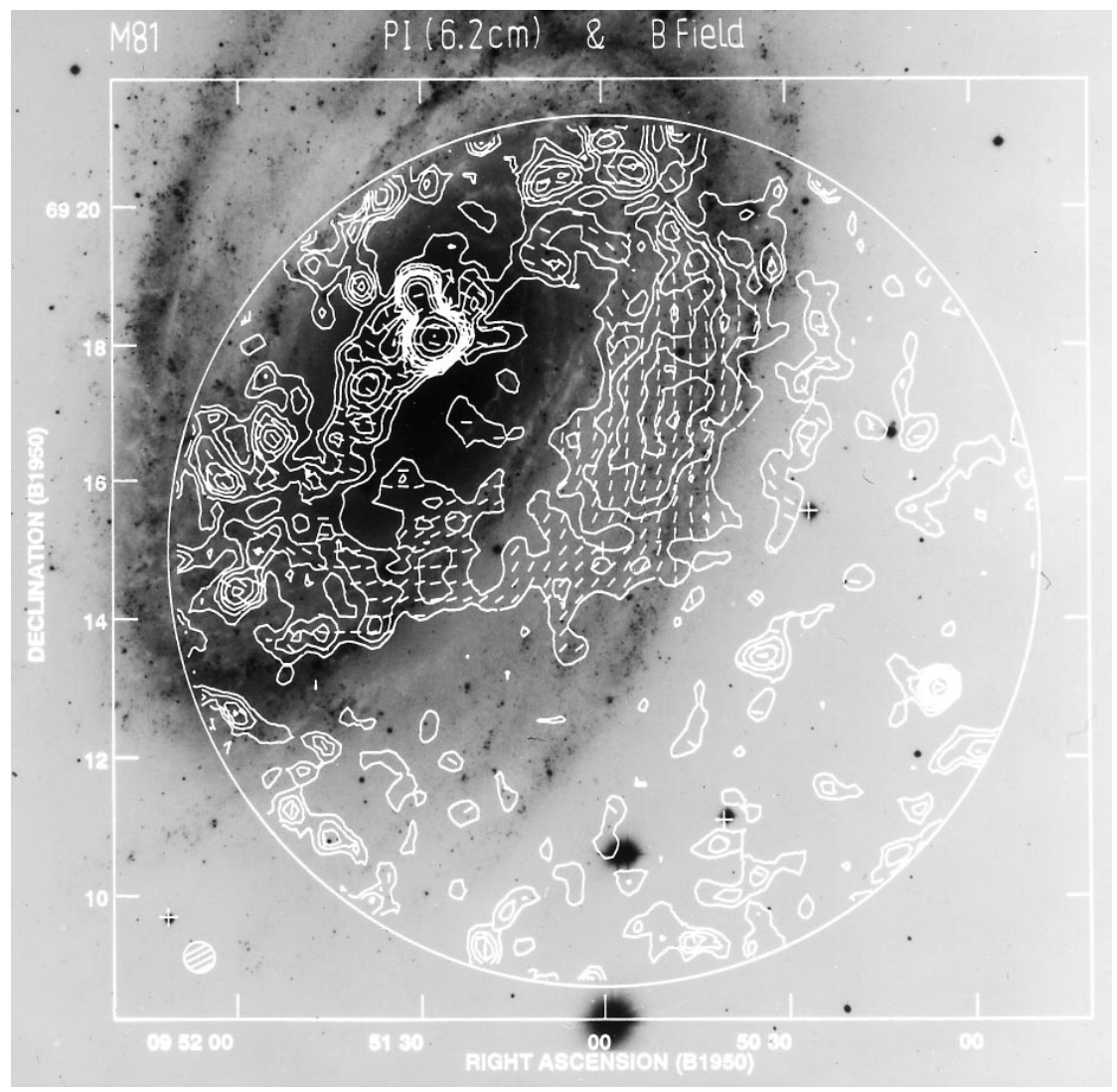

Figure 2 Polarized synchrotron intensity (contours) and magnetic field orientation in the southwestern part of M81 (obtained by rotating the $E$-vectors by $90^{\circ}$ ), observed at $\lambda 6.2 \mathrm{~cm}$ with the VLA ( 25 arcsec synthesized beam). The circle indicates the half-power diameter of the primary beam. (From Schoofs 1992.) 


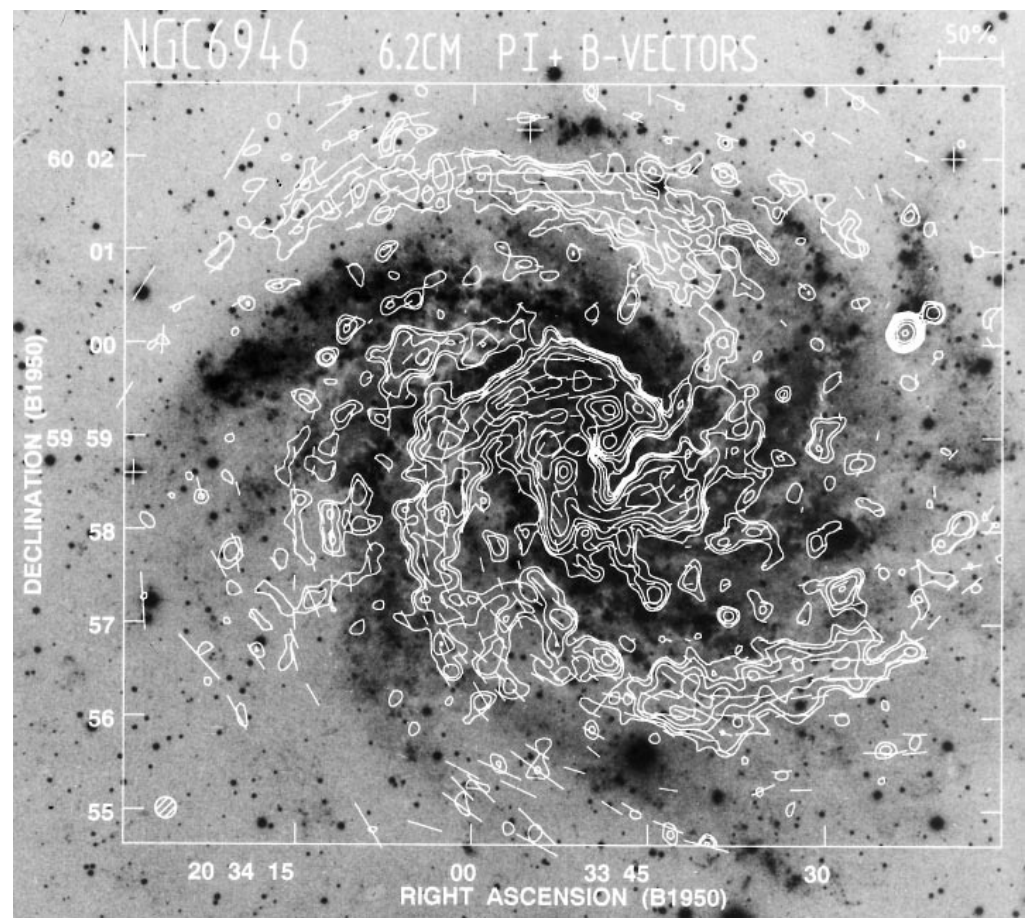

Figure 3 Polarized synchrotron intensity (contours) and magnetic field orientation of NGC 6946 (obtained by rotating $E$-vectors by $90^{\circ}$ ) observed at $\lambda 6.2 \mathrm{~cm}$ with the VLA (12.5 arcsec synthesized beam) and combined with extended emission observed with the Effelsberg 100-m telescope (2.5 arcmin resolution). The lengths of the vectors are proportional to the degree of polarization. (From Beck \& Hoernes 1996.)

gas is exceptionally low. Subtraction of the diffuse, unpolarized background gives a degree of polarization of 30-65\%, with the implication that the fields in the magnetic spiral arms must be almost totally aligned with the optical arms.

\subsection{ASS, BSS, or What?}

The available data on global magnetic structures in spiral galaxies are compiled in Tables 2 and 3. Most of the results were obtained using the RM analysis method (see Section 2.2); the more advanced $\psi$-analysis method has as yet only been applied to M51 (and in simplified form to M31, IC 342, and M81).

Singly-periodic RM variations indicative of ASS have been detected in the disks of M31 (Sofue \& Takano 1981, Beck 1982) and IC 342 (Gräve \& Beck 1988, Krause et al 1989a, Sokoloff et al 1992). In M31 Ruzmaikin et al (1990) found evidence for the presence of the $m=1$ mode at lower amplitude in the 
Table 2 Magnetic field structures of normal galaxies with low or moderate inclination as derived from synchrotron polarization data

\begin{tabular}{|c|c|c|c|}
\hline Galaxy & $\begin{array}{l}\text { Instrument }{ }^{\mathrm{a}} \text { and } \\
\text { wavelength }\end{array}$ & Field structure & References \\
\hline Milky Way & & ASS? & See Section 3.8 \\
\hline M31 & $\begin{array}{l}\text { E } 11,6 \mathrm{~cm} \\
\text { V } 20,6 \mathrm{~cm}\end{array}$ & ASS (with weaker BSS) & $\begin{array}{l}\text { Beck (1982), } \\
\text { Ruzmaikin et al (1990) }\end{array}$ \\
\hline M33 & $\mathrm{E} 21,18,11,6,2.8 \mathrm{~cm}$ & Spiral (BSS?) & Buczilowski \& Beck (1991) \\
\hline M51 & $\begin{array}{l}\text { E } 6,2.8 \mathrm{~cm} \\
\text { V } 20,18,6 \mathrm{~cm} \\
\text { W } 21,6 \mathrm{~cm}\end{array}$ & $\begin{array}{l}\text { MSS, } \\
\text { magnetoionic halo }\end{array}$ & $\begin{array}{l}\text { EM Berkhuijsen et al, in prep., } \\
\text { Horellou et al (1992), } \\
\text { Segalovitz et al (1976) }\end{array}$ \\
\hline M81 & $\begin{array}{l}\text { E } 6,2.8 \mathrm{~cm} \\
\text { V } 20,6 \mathrm{~cm}\end{array}$ & BSS (with weaker ASS) & $\begin{array}{l}\text { Krause et al (1989b), } \\
\text { Sokoloff et al (1992) }\end{array}$ \\
\hline M83 & $\begin{array}{l}\text { E } 6,2.8 \mathrm{~cm} \\
\text { V } 20,6 \mathrm{~cm} \\
\text { A } 13 \mathrm{~cm}\end{array}$ & Spiral and $\|$ bar & $\begin{array}{l}\text { Neininger et al (1993), } \\
\text { Sukumar \& Allen (1989), } \\
\text { Ehle (1995) }\end{array}$ \\
\hline M101 & E $6 \mathrm{~cm}$ & Spiral & Gräve et al (1990) \\
\hline SMC & $\mathrm{P} 21,12 \mathrm{~cm}$ & $\|$ main ridge & Haynes et al (1986) \\
\hline LMC & $\mathrm{P} 21,12,6 \mathrm{~cm}$ & Loop south of 30 Dor & Klein et al (1993) \\
\hline IC 342 & $\begin{array}{l}\text { E } 11,6 \mathrm{~cm} \\
\text { V } 20,6,3.5 \mathrm{~cm}\end{array}$ & $\begin{array}{l}\text { ASS, } \\
\text { magnetic spiral arms }\end{array}$ & $\begin{array}{l}\text { Sokoloff et al (1992), } \\
\text { Krause et al (1989a) }\end{array}$ \\
\hline NGC 1566 & A $20,13,6 \mathrm{~cm}$ & Spiral & Ehle et al (1996) \\
\hline NGC 1672 & A $20,13,6 \mathrm{~cm}$ & Spiral & M Ehle et al (in prep.) \\
\hline NGC 2276 & $\mathrm{~V} 20,6 \mathrm{~cm}$ & Spiral (BSS?) & Hummel \& Beck (1995) \\
\hline NGC 2903 & $\begin{array}{l}\text { E } 6,2.8 \mathrm{~cm} \\
\text { V } 18,20 \mathrm{~cm}\end{array}$ & Spiral & R Beck et al (in prep.) \\
\hline NGC 3627 & E $2.8 \mathrm{~cm}$ & $\|$ dust lane & M Urbanik et al (in prep.) \\
\hline NGC 4038/39 & V $20,6,3.6 \mathrm{~cm}$ & $\|$ tidal arm & E Hummel \& R Beck (in prep.) \\
\hline NGC 4254 & $\mathrm{E} 6,2.8 \mathrm{~cm}$ & \| compression region & Soida et al (1996) \\
\hline NGC 4258 & $\begin{array}{l}\text { E } 6,2.8 \mathrm{~cm} \\
\text { W } 49,21 \mathrm{~cm} \\
\text { V } 20,6 \mathrm{~cm}\end{array}$ & $\|$ anomalous arms & Hummel et al (1989) \\
\hline NGC 4449 & E $2.8 \mathrm{~cm}$ & \| HII chains & U Klein et al (submitted) \\
\hline NGC 5055 & E $2.8 \mathrm{~cm}$ & $\|$ disk & M Soida et al (in prep.) \\
\hline NGC 6946 & $\begin{array}{l}\text { E } 6,2.8 \mathrm{~cm} \\
\text { V } 20,18,6,3.5 \mathrm{~cm}\end{array}$ & $\begin{array}{l}\text { Spiral (MSS?), } \\
\text { magnetic spiral arms }\end{array}$ & $\begin{array}{l}\text { Ehle \& Beck (1993), } \\
\text { Beck \& Hoernes (1996) }\end{array}$ \\
\hline
\end{tabular}

${ }^{\mathrm{a}}$ Instruments: $\mathrm{E}=$ Effelsberg 100-m, $\mathrm{A}=$ Australia Telescope Compact Array, $\mathrm{P}=$ Parkes $64-\mathrm{m}, \mathrm{W}=$ Westerbork Synthesis Radio Telescope, $\mathrm{V}=$ Very Large Array. 
Table 3 Magnetic field structures of (almost) edge-on galaxies

\begin{tabular}{|c|c|c|c|}
\hline Galaxy & $\begin{array}{l}\text { Instrument }{ }^{a} \text { and } \\
\text { wavelength }\end{array}$ & Field structure & References \\
\hline M82 & V $6,3.6$ & Radial & Reuter et al (1994) \\
\hline NGC 253 & $\begin{array}{l}\text { P } 6 \mathrm{~cm} \\
\text { V } 20,6 \mathrm{~cm} \\
\text { E } 2.8 \mathrm{~cm}\end{array}$ & \| plane (ASS?) & $\begin{array}{l}\text { Harnett et al (1990), } \\
\text { Carilli et al (1992), } \\
\text { Beck et al (1994b) }\end{array}$ \\
\hline NGC 891 & $\begin{array}{l}\text { V } 20,6 \mathrm{~cm} \\
\text { E } 2.8 \mathrm{~cm}\end{array}$ & $\| \&$ inclined to plane & $\begin{array}{l}\text { Sukumar \& Allen (1991), } \\
\text { Dumke et al (1995) }\end{array}$ \\
\hline NGC 1808 & $\mathrm{~V} 20,6 \mathrm{~cm}$ & Extensions $\perp$ plane & Dahlem et al (1990) \\
\hline NGC 3628 & $\begin{array}{l}\text { V } 20,6 \mathrm{~cm} \\
\text { E } 2.8 \mathrm{~cm}\end{array}$ & $\| \&$ inclined to plane & $\begin{array}{l}\text { Reuter et al (1991), } \\
\text { Dumke et al (1995) }\end{array}$ \\
\hline NGC 4565 & $\begin{array}{l}\text { V } 20,6 \mathrm{~cm} \\
\text { E } 2.8 \mathrm{~cm}\end{array}$ & \| plane & $\begin{array}{l}\text { Sukumar \& Allen (1991), } \\
\text { Dumke et al (1995) }\end{array}$ \\
\hline NGC 4631 & $\begin{array}{l}\text { E } 6,2.8 \mathrm{~cm} \\
\text { V } 20,6,3.5 \mathrm{~cm}\end{array}$ & $\begin{array}{l}\perp \text { plane (inner region), } \\
\| \& \text { inclined to plane (outer regions) }\end{array}$ & $\begin{array}{l}\text { Hummel et al (1991a), } \\
\text { Golla \& Hummel (1994) }\end{array}$ \\
\hline NGC 4945 & P $6 \mathrm{~cm}$ & Extensions $\perp$ plane & Harnett et al (1989), \\
\hline NGC 5775 & V $6 \mathrm{~cm}$ & \| plane & Golla \& Beck (1990) \\
\hline NGC 5907 & V $6 \mathrm{~cm}$ & \| plane & M Dumke \& M Krause (in prep.) \\
\hline NGC 7331 & $\begin{array}{l}\text { E } 2.8 \mathrm{~cm} \\
\text { V } 20,6 \mathrm{~cm}\end{array}$ & Almost \| plane & $\begin{array}{l}\text { Dumke et al (1995), } \\
\text { E Hummel (unpubl.) }\end{array}$ \\
\hline Circinus & A $13,6 \mathrm{~cm}$ & $\perp$ northern plume & Elmouttie et al (1995) \\
\hline
\end{tabular}

anstruments: E = Effelsberg 100-m, A = Australia Telescope Compact Array, $\mathrm{P}=$ Parkes 64-m, $\mathrm{W}=$ Westerbork Synthesis Radio Telescope, $\mathrm{V}=$ Very Large Array.

outer regions, superimposed on the dominating $m=0$ mode. In NGC 6946 the phase of the azimuthal RM variation differs significantly from the value of the mean magnetic pitch angle (Ehle \& Beck 1993). Recent high-resolution data for this galaxy (R Beck \& P Hoernes, in preparation) indicate some correlation of RM with the optical spiral arms, suggesting local enhancements of RM due to thermal gas, rather than to field geometry. The magnetic spiral arms (where thermal gas density is low) seem to have RMs of opposite sign (R Beck \& $\mathrm{P}$ Hoernes, in preparation), indicative of the $m=0$ mode or, more realistically, a superposition of the $m=0$ and the $m=2$ mode with about equal amplitudes. In NGC 253, seen almost edge-on, the large-scale magnetic field has opposite directions on the "left" and "right" of the rotation axis of the inner disk. NGC 253 is thus another candidate for an ASS disk field (Beck et al 1994b).

The only clear candidate for a BSS symmetry is M81 (Krause et al 1989b, Sokoloff et al 1992). The analysis of Krause et al (1989b) indicated that the 
magnetic neutral lines are in the interarm space. In M33 the weak polarized emission leads to large uncertainties in RM, and a bisymmetric field can be claimed only with some caution (Buczilowski \& Beck 1991). The same is true for NGC 2276 (Hummel \& Beck 1995). The galaxies M33, M81, and NGC 2276 show signs of gravitational interaction, which can be important in producing nonaxisymmetric dynamo fields (see Sections 6.2 and 8.2). Thus these are all candidates for MSS status. Other claims of a dominating bisymmetric field (Sofue et al 1985, 1986) are of much lower significance.

The strongly interacting galaxy M51 is a special case where the pitfalls of data interpretation can be demonstrated. M51 was thought to contain a bisymmetric field (Tosa \& Fujimoto 1978, Horellou et al 1990), but this was not confirmed by later Effelsberg and VLA measurements. At $\lambda \geq 10 \mathrm{~cm}$, Faraday depolarization is strong and the observed polarized emission originates in the upper disk or halo (Horellou et al 1992). By analyzing all available data in terms of the $\psi$ angles, EM Berkhuijsen et al (in preparation) have found that the field in M51 can be described as MSS, with axisymmetric and bisymmetric components having about equal weights in the disk, together with a horizontal axisymmetric halo field with opposite direction.

The RM variation in M83 is doubly-periodic (Neininger et al 1993), but the phase is inconsistent with BSS symmetry. A future analysis of polarization angles including recent observations at $\lambda 13 \mathrm{~cm}$ (Ehle 1995) might clarify the case. The RM pattern in M83 indicates a nonaxisymmetric distribution of gas or velocity field, an MSS field, or both.

\subsection{Magnetic Fields in Galactic Halos}

Vertical dust lanes are often seen in edge-on galaxies, which may indicate vertical magnetic field lines (Sofue 1987). Their initial detection via polarized radio emission in NGC 4631 by Hummel et al (1988a) prompted a systematic search in several nearby edge-on galaxies. Radio halos were detected also in NGC 253 (Carilli et al 1992) and in NGC 4666 (M Dahlem et al, in preparation). A survey of 181 edge-on galaxies observed with the Effelsberg and VLA radio telescopes (Hummel et al 1991b) disclosed no other cases with pronounced halos.

In contrast, NGC 891 (Hummel et al 1991a), NGC 3628 (Reuter et al 1991), NGC 5775 (Golla \& Beck 1990), and most other edge-on galaxies (Hummel 1990) do not possess extended radio halos. These galaxies have thick disks with typical synchrotron scale heights of $\simeq 1 \mathrm{kpc}$. In most of these galaxies the observed field orientations are approximately parallel to the disk (Dumke et al 1995; see also Table 3). The same is true for NGC 4945 (Harnett et al 1989) and NGC 1808 (Dahlem et al 1990), but there the polarized emission is restricted to two localized regions, one on each side of the plane. In the disks themselves, the polarized emission at $\lambda \geq 6 \mathrm{~cm}$ is weak due to Faraday depolarization. 
The other extremes are NGC 4565 (Sukumar \& Allen 1991) and M31. The radio emission from any thick disk of M31 is not detectable and must be at least 200 times weaker than for NGC 891 (Berkhuijsen et al 1991). Either the low star-formation rates in M31 and NGC 4565 are below the threshold for the chimney-type outflows (Dahlem et al 1995) or the dynamo does not operate in the halos of these galaxies.

The increase of the degree of polarization with height above the disk of NGC 891 has been analyzed by Hummel et al (1991a). The data can be well modeled by Faraday depolarization in a thermal gas of scale height $\simeq 1 \mathrm{kpc}$ together with a turbulent magnetic field of scale height $\simeq 4 \mathrm{kpc}$. The scale height of the thermal gas as derived from the radio data agrees well with that observed in $\mathrm{H} \alpha$ (Rand et al 1990, Dettmar 1990). The scale height of the turbulent halo field is consistent with equipartition between the field and cosmic-ray energy densities, where $z_{\mathrm{b}}=2 z_{\mathrm{CR}}=\left(3+\alpha_{\mathrm{s}}\right) z_{\mathrm{syn}} \simeq 3.6 \mathrm{kpc}$ for a synchrotron scale height of $z_{\text {syn }} \simeq 0.9 \mathrm{kpc}$ and $\alpha_{\mathrm{s}} \simeq 1.0$ (Hummel et al 1991a).

NGC 253 is the edge-on galaxy with the brightest and largest halo observed so far (Carilli et al 1992), extending to at least $9 \mathrm{kpc}$ above the plane. It also has the brightest X-ray halo (Pietsch 1994), so that a strong outflow from the disk or the nucleus driven by the high star-formation rate seems probable. Gas outflow from the nucleus has indeed been found (Dickey et al 1992). Nevertheless, the regular magnetic field is predominantly parallel to the plane in the disk and in the halo (Beck et al 1994b; see Figure 4), possibly due to strong differential rotation even near the center.

NGC 4631 is another rare case of an extended radio halo, possibly driven by a strong galactic wind. The synchrotron scale height of $\simeq 2 \mathrm{kpc}$ is twice as large as for the bulk of edge-on galaxies (Hummel 1990). The magnetic field lines are roughly perpendicular to the inner disk, which is almost rigidly rotating (Hummel et al 1991a, Golla \& Hummel 1994). In this respect, NGC 4631 is exceptional compared with most edge-on galaxies. NGC 4631 also shows signs of gravitational interaction. A few regions with field orientations parallel to the disk are visible in the (differentially rotating) outer disk.

A striking case of a strong galactic wind is M82, which has quasi-radial field lines (Reuter et al 1994). Even a field of $\simeq 50 \mu \mathrm{G}$ strength (Klein et al 1988) cannot resist the flow with a velocity in excess of $1000 \mathrm{~km} \mathrm{~s}^{-1}$.

Vertical magnetic fields may be a result of disk-halo interactions. The Parker instability produces alternating vertical magnetic fields. A vertical galactic wind of speed $V_{z}$ could also drag the field from the disk. However, an azimuthal gradient of $V_{z}$ is required to produce $B_{z}$ from $B_{\phi}$ and a radial gradient of $V_{z}$ to obtain $B_{z}$ from $B_{r}$ (see Section 7). 
Rotation measures in galactic halos are important for revealing the direction of the field and thus its parity with respect to the midplane. Golla \& Hummel (1994) could not find a clear RM pattern from their data of NGC 4631. Beck et al (1994b) determined rotation measures at a few positions in the lower halo of NGC 253 and found weak evidence for RMs of the same sign at $\simeq 5 \mathrm{kpc}$ above and below the plane, as expected for an even-parity mode.

In face-on galaxies "coronal holes" have been observed as regions of high rotation measure (with ensuing higher depolarization) with neither enhanced plasma density ( $\mathrm{H} \alpha$ or X-ray emission) nor enhanced total field strength (total synchrotron emission). In these regions magnetic lines probably open into the halo. The RM maps of IC 342 (Krause et al 1989a) and NGC 6946 (Beck 1991) seem to show such phenomena. The rotation measures in NGC 6946, determined between $\lambda 18 \mathrm{~cm}$ and $\lambda 20 \mathrm{~cm}$, are small and almost constant, except

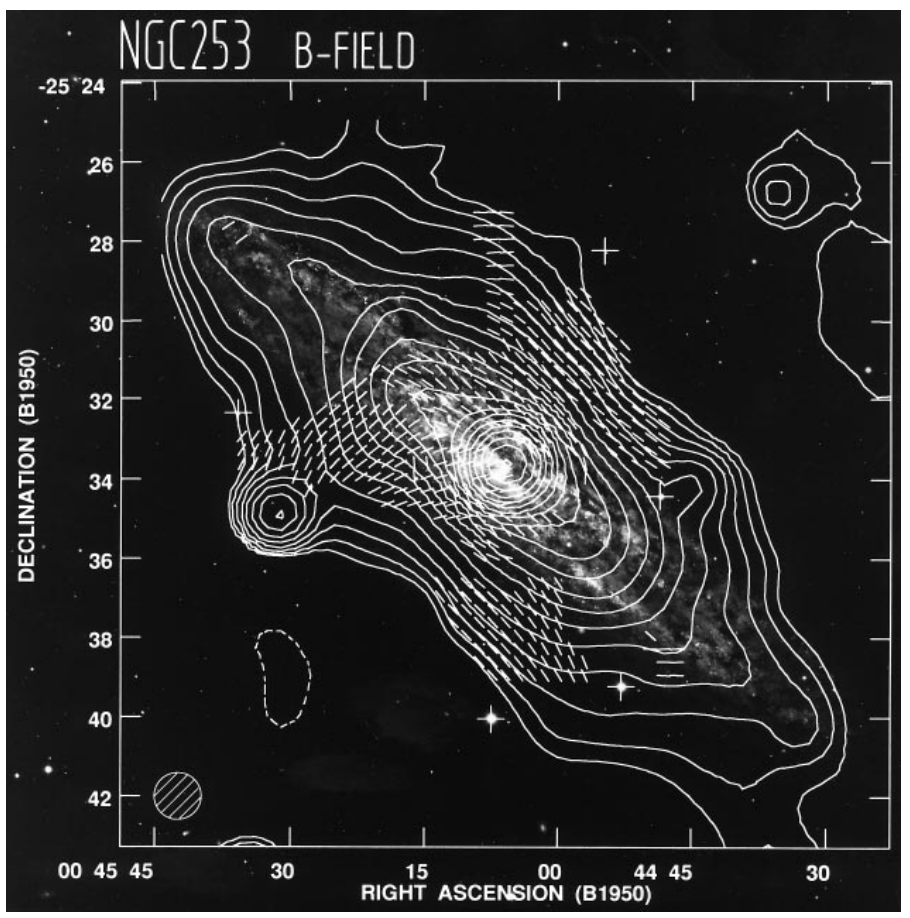

Figure 4 Total radio intensity (contours) and magnetic field orientation of NGC 253 (obtained by rotating $E$-vectors by $90^{\circ}$ ), observed at $\lambda 2.8 \mathrm{~cm}$ with the Effelsberg telescope (disk field) and at $\lambda 20 \mathrm{~cm}$ wavelength with the VLA (halo field). The resolution is 70 arcsec. (From Beck et al 1994b.) 
in the southwest quadrant, where both high and low values occur in a region of $\simeq 10 \mathrm{kpc}$ in extent (Beck 1991). The spiral arms in the southwest quadrant of NGC 6946 are more diffuse and the X-ray emission is weaker (Schlegel 1994) than in the remainder of the galaxy. Thus galactic coronal holes may occur in regions of low star-forming activity.

\subsection{Magnetic Fields in High-Redshift Galaxies}

It is likely that spiral galaxies have possessed their large-scale magnetic fields at least $6 \times 10^{9} \mathrm{yr}$ ago (corresponding to a redshift $z \simeq 0.5$ ) (Kronberg 1994, Perry 1994). The most convincing evidence is the detection of Faraday rotation attributed to a galaxy at $z=0.395$ (Kronberg et al 1992). The inferred largescale magnetic field strength is $1-4 \mu \mathrm{G}$ and its direction reverses on a scale of $\simeq 3 \mathrm{kpc}$. Kronberg et al (1992) argue for a bisymmetric magnetic structure, but this may equally well be an axisymmetric field with reversals (Poezd et al 1993).

Statistical studies of quasar samples (Kronberg \& Perry 1982, Welter et al 1984, Perry et al 1993) indicate that excess Faraday rotation correlates with the presence of intervening absorbers. The size of the absorbers has been estimated as $45 \mathrm{kpc}$, with their global magnetic fields of 2-10 $\mu \mathrm{G}$; these are probably galactic disks and/or halos. Wolfe et al (1992) and Oren \& Wolfe (1995) have argued that damped Ly $\alpha$ systems [i.e. putative young galactic disks (Wolfe 1988, Wolfe et al 1993)] possess $\mu \mathrm{G}$-strength global magnetic fields at $z \simeq 1-2$ when they are only 1-3 Gyr old. (However, statistical analyses of this kind are extremely difficult, in particular because of poor statistics, different selection effects, complications in isolating contributions of other intervenors such as our Galaxy, galaxy clusters, etc (Perry et al 1993, Perry 1994). The earliest time at which galaxies possess their large-scale magnetic fields still has to be established. Theoretical models of magnetic fields in young galaxies are discussed in Section 5.3.

A straightforward implication of these studies is a lower limit on the seed magnetic field required for galactic dynamos. If an $\Omega=1$ cosmology is assumed, then this limit is $2 \times 10^{-18} \mathrm{G}$ (Kronberg et al 1992), or even possibly $10^{-9}-10^{-11} \mathrm{G}$ if a tentative identification of excess RM in the quasar $1331+170$ with an absorber at $z=1.775$ is confirmed (Perry 1994) (see also Section 5).

\subsection{The Milky Way}

Observations in the Milky Way offer a unique opportunity for studying interstellar magnetic fields in a detail unobtainable for even nearby external galaxies. However, the plethora of local detail, which obscures any grand-design features of the magnetic field in the Milky Way, still prevents a reliable picture from being obtained. 
3.8.1 MAGNETIC fiELD IN THE SOLAR VICINITY The most reliable estimates concerning the large-scale magnetic field near the Sun are obtained from statistical analyses of Faraday rotation measures of nearby pulsars (within 2-3 kpc from the Sun) and high-latitude extragalactic radio sources, because larger samples involve lines of sight passing through remote regions in the Galaxy for which the inferred magnetic field configuration is less reliable (see SimardNormandin \& Kronberg 1980, Rand \& Kulkarni 1989, and references therein). The regular field strength is $\simeq 2 \mu \mathrm{G}$, probably stronger within the arm.

The field is directed towards a galactic longitude of about $90^{\circ}$ (see, e.g. Ruzmaikin et al 1978, Rand \& Lyne 1994), with an accuracy of 10-20 . The scatter between different determinations makes it difficult to say whether it is aligned with the local spiral arm (pitch angle of about $-15^{\circ}$ ) or not. A tentative upper limit on the magnetic pitch angle, $|p| \leq 15^{\circ}$, implies that $\left|\bar{B}_{r}\right| \leq 0.3\left|\bar{B}_{\phi}\right|$.

The best agreement with observations is provided by models with the horizontal global magnetic field similarly directed above and below the midplane (S-type field) (Gardner et al 1969; Vallée \& Kronberg 1973, 1975). Claims of an odd symmetry (Morris \& Berge 1964; Andreassian 1980, 1982) probably result from contamination by strong local distortions in the magnetic field. A similar problem prevents the reliable detection of the vertical magnetic field $\bar{B}_{z}$ near the Sun: It is so weak that it cannot be separated from local magnetic inhomogeneities, $\left|\bar{B}_{z}\right| \ll\left|\bar{B}_{r}\right|,\left|\bar{B}_{\phi}\right|$.

Because the warm interstellar medium is the main contributor to the electron density in the diffuse ISM, rotation measures sample mainly this phase of the ISM. Heiles (1996) has argued that the warm interstellar medium occupies only $\simeq 20 \%$ of the total volume in the Milky Way, so that the resulting $\bar{B}$ does not reflect the true volume-averaged field. This argument would apply also to external galaxies, where Faraday rotation is also used to study $\bar{B}$. However, the observed coherency of RM patterns over large regions in many nearby galaxies indicates that the inferred magnetic field is global rather than restricted to a small fraction of the volume (see also Section 3.1).

3.8.2 REVERSALS OFTHE MAGNETIC fiELD AND ITS AZIMUTHAL STRUCTURE The property of the magnetic field in the Milky Way that distinguishes it from probably most other galaxies investigated up to now is the reversals of the regular field along the radius. The reversal closest to the Sun between the local (Orion) and the next arm to the center (Sagittarius) was first detected by Simard-Normandin \& Kronberg (1979). The reversal is located in the interarm region at about 0.4$0.5 \mathrm{kpc}$ inside the solar circle (see Rand \& Lyne 1994).

There are some indications of more reversals at both smaller and larger galactocentric distances, but this evidence is much more controversial because distant spiral arms occupy smaller areas on the sky. Simard-Normandin \& 
Kronberg (1980) and Vallée (1983) argued that there is no reversal between the local and the next outer (Perseus) arms, whereas other authors found some evidence for an outer reversal (Agafonov et al 1988, Rand \& Kulkarni 1989, Lyne \& Smith 1989, Clegg et al 1992). Two additional reversals were claimed for the inner Galaxy by Sofue \& Fujimoto (1983) and Han \& Qiao (1994), but most analyses more conservatively imply only one more, at a galactocentric radius of $5.5 \mathrm{kpc}$ (Vallée et al 1988, Vallée 1991, Rand \& Lyne 1994). The controversy about the number of reversals is partly due to difficulties in the analysis of Faraday rotation measures. There are natural complications associated with strong local distortions of magnetic field, e.g. the North Polar Spur or the Gum Nebula. However, there are also many pitfalls in the statistical analyses. Many results rely on simple "naked-eye" fitting of the observational data (e.g. Simard-Normandin \& Kronberg 1980, Sofue \& Fujimoto 1983), which is especially dangerous when the global structure is investigated; others are based on nonrigorous applications of statistical tests (e.g. Han \& Qiao 1994). Vallée (1996) discusses some of these problems. More rigorous studies imply an axisymmetric field with two reversals (Rand \& Kulkarni 1989, Rand \& Lyne 1994), although more cannot be excluded. The radial distribution of the magnetic field strength is shown in Figure 5 (see also Heiles 1996).

The available statistical analyses adopt either a bisymmetric structure of the global magnetic field (Simard-Normandin \& Kronberg 1980, Sofue \& Fujimoto 1983, Han \& Qiao 1994) or a concentric-ring model in which magnetic field lines are directed exactly in the azimuthal direction. Comparison between these two models often shows that the latter provides a better fit to the data (e.g. Rand \& Kulkarni 1989); however, the concentric-ring model is unrealistically simplistic. The regular magnetic field cannot have a zero pitch angle everywhere (see Section 8.3), even if it does near the Sun. The model is consistent neither with theoretical ideas about galactic magnetic fields nor with observations of external galaxies (Section 3.3). The pitch angle of the magnetic field should be a model parameter, possibly a function of position, obtained from fits to data rather than fixed to be zero (or any other value) beforehand. Another problem is that the magnetic field may really correspond to a superposition of different azimuthal modes, so that attempts at fitting a purely axisymmetric or bisymmetric model may lead to erroneous results.

The presence of reversals in the Milky Way is often interpreted as an unambiguous indication of the bisymmetric global structure of the magnetic field. As we discuss in Section 8.5, axisymmetric magnetic structures may also contain reversals, and mean-field dynamo models for the Milky Way favor an axisymmetric field structure. 


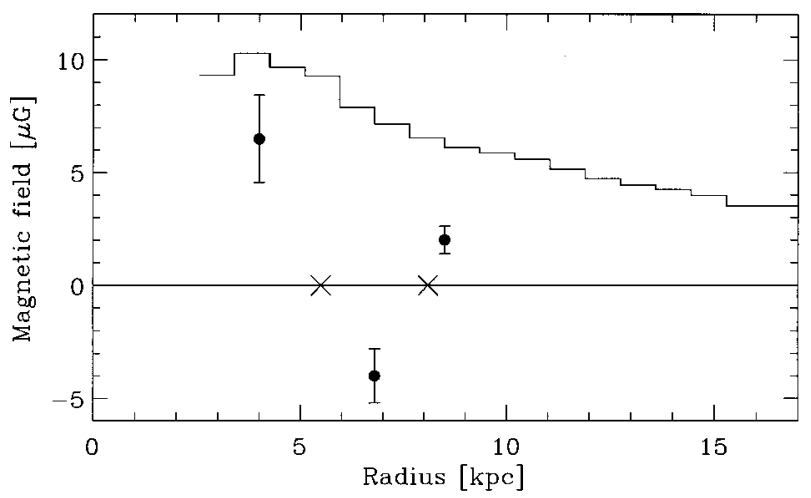

Figure 5 The strength of the large-scale magnetic field in the Milky Way (full circles with error bars) and positions of its reversals (crosses), as inferred from pulsar rotation measures (Rand \& Lyne 1994). Note a gradual increase of $|\bar{B}|$ towards smaller radii (a positive $\bar{B}$ corresponds to the field direction towards the first and second Galactic quadrants). Error bars shown correspond to $30 \%$ uncertainty and are chosen tentatively to indicate a scatter of the available estimates at $r=8.5 \mathrm{kpc}$, the Galactic radius of the sun. The solid line shows the strength of the total magnetic field, averaged in azimuth as obtained by EM Berkhuijsen (in preparation) from the deconvolved surface brightness of synchrotron emission at $408 \mathrm{MHz}$ (Beuermann et al 1985), assuming energy equipartition between magnetic field and cosmic rays; the accuracy of this estimate is probably $\simeq 30 \%$.

Field reversals have rarely been observed in external galaxies, only in BSS candidates (see Table 2) and possibly in a galaxy at redshift 0.395 (Kronberg et al 1992; see Section 3.7). In some galaxies, the resolution of the observations is high enough to detect reversals if they were present: This is the case for M31 observed with a resolution of $\simeq 1 \mathrm{kpc}$ near the major axis (Beck 1982, Ruzmaikin et al 1990). In other galaxies the resolution of Faraday rotation data is lower (e.g. Krause et al 1989a, Buczilowski \& Beck 1991, Ehle \& Beck 1993) and reversals cannot be excluded. However, the number of reversals within the telescope beam cannot be large as this would average out any Faraday rotation.

Because the Sun is located fairly close to a reversal, the strength of the regular magnetic field at $r \simeq 8.5 \mathrm{kpc}$ is less likely to be a representative value for the bulk of spiral galaxies or even for the Milky Way itself. Values of order 4-6 $\mu \mathrm{G}$ seem to be more typical.

\section{GALACTIC DYNAMO THEORY}

We now discuss the mechanisms generating large-scale fields that have been presented in the previous section. We begin by considering first the small-scale magnetic fields. 


\subsection{Random Magnetic Fields}

The interstellar medium is turbulent and thus any embedded magnetic field must have a random small-scale component. The presence of this component is crucial in all theories of large-scale dynamo action. There are several mechanisms that produce fluctuations in the interstellar magnetic fields: (a) tangling of the large-scale field by turbulence and from Parker and thermal instabilities, (b) compression of ambient magnetic fields by shock fronts associated with supernova remnants and stellar winds, and $(c)$ self-generation of random magnetic fields by turbulence (small-scale dynamo). All of these mechanisms act together, and each imprints its own statistical properties onto the magnetic fields.

The available observational and theoretical knowledge of random magnetic fields and their maintenance in the ISM is rather poor. Instead, crude descriptions in terms of global quantities such as mean magnetic energy are usually applied. A widely used concept is that of equipartition between the magnetic and kinetic energy in the turbulence (Kraichnan 1965, Zweibel \& McKee 1995), which implies that the rms random magnetic field strength is given by $b \simeq B_{\text {eq }} \equiv\left(4 \pi \rho v^{2}\right)^{1 / 2}$, with $v$ the rms turbulent velocity and $\rho$ the density. The equipartition value is significant in that the Lorentz force is expected to become comparable to the forces driving the turbulent flow as equipartition is approached. (This $B_{\text {eq }}$ is not to be confused with the equipartition field strength in Sections 2 and 3, where equipartition refers to the estimated cosmic-ray energy density used to deduce the field strength from the synchrotron emission.) Interstellar turbulence is often treated as an ensemble of random Alfvén waves (McIvor 1977, Ruzmaikin \& Shukurov 1982, McKee \& Zweibel 1995) for which the equipartition holds exactly. Magnetic fluctuations are accompanied also by fluctuations in density (Armstrong et al 1995), so that other mechanisms, possibly nonpropagating fluctuations, must contribute to the interstellar turbulence (Higdon 1984). The random magnetic fields in the Milky Way are typically 4-6 $\mu \mathrm{G}$ (Ohno \& Shibata 1993), close to $B_{\text {eq }}$.

Another component of the random magnetic field, one that is associated with interstellar (super) bubbles, is observed in the Milky Way (Heiles 1989, Heiles et al 1993, Vallée 1993). The magnetic field in HI shells, detected via the Zeeman effect, seems to be concentrated in filaments with the magnetic pressure larger than the gas pressure. The field strength in magnetic bubbles around OB associations, as obtained from Faraday rotation measurements, follows the density dependence $b \propto \rho$ expected for a shocked medium.

The small-scale dynamo (Kazantsev 1968, Meneguzzi et al 1981) must be an important source of interstellar random magnetic fields (Sokoloff et al 1990). A distinctive feature of this component of the interstellar field, item $(b)$ above, 
is that it is organized in intermittent magnetic ropes of small filling factor and lengths comparable to the correlation length of the turbulence $(50-100 \mathrm{pc})$. The rms strength of the magnetic fluctuations generated by this mechanism is possibly close to the equipartition value, but the field within the filaments may be significantly higher (Belyanin et al 1993). For example, three-dimensional simulations of convective small-scale dynamo action at magnetic Reynolds numbers of about 1000 (Nordlund et al 1992) give $B_{\mathrm{rms}}=0.4 B_{\mathrm{eq}}$ and $B_{\mathrm{max}}=$ $3 B_{\text {eq. }}$. We note that in the interstellar gas of elliptical galaxies a small-scale dynamo may be the only source of magnetic fields, generating random fields of $\mu \mathrm{G}$ strength and a few hundred parsecs in scale (Moss \& Shukurov 1996).

\subsection{Large-Scale Fields}

The main challenge in the theory of galactic magnetism is to explain the origin and structure of the observed large-scale field. In Figure 6 we sketch different routes by which large-scale magnetic fields may arise. Large-scale flows (shear, compression) together with turbulence effects (swirling motions and inverse cascade - see below) can amplify weak seed magnetic fields (Section 5), thereby converting small-scale fields into large-scale fields. The amplifying effect of swirling motions on the large-scale field is described by the $\alpha$-effect (Parker 1955, Steenbeck et al 1966, Moffatt 1978). Such motions also lead to an inverse cascade from the conservation properties of the magnetic helicity (Frisch et al 1975, Pouquet et al 1976) and from the cross-helicity effect (Yoshizawa \& Yokoi 1993).

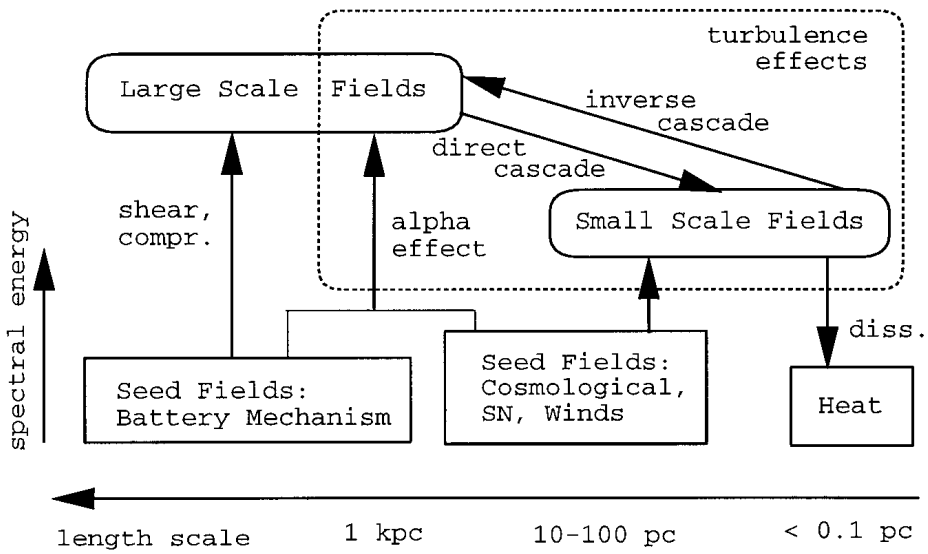

Figure 6 Sketch illustrating the various routes by which large-scale magnetic fields can arise. Turbulence effects (inverse cascade and $\alpha$-effect) combined with shear and compression (differential rotation) amplify weak magnetic fields to produce strong large-scale fields. 
These concepts were originally applied to stellar turbulence, where the existence of dynamos can almost be considered as an observational fact. It is not clear, however, how much galactic turbulence has in common with thermal turbulence in stars. Nevertheless, statistical properties of turbulence in molecular clouds seem to be remarkably similar to those determined from numerical simulations of ordinary compressible turbulence (Falgarone et al 1994).

There have been attempts to explain the large-scale magnetic field without invoking dynamo action. The turbulence must then be regarded as unimportant, and a large-scale seed magnetic field has to be amplified by large-scale shearing and compression alone. The inevitable eventual decay is assumed then to be considerably postponed (e.g. Kulsrud 1986). A model of this type has recently been proposed by Chiba \& Lesch (1994), who consider fields that are maintained by an unspecified mechanism at large distances. Because these processes describe field amplification by shearing and compression alone, it is quite uncertain whether they can give fields of the strength and topology required at ages of about $10 \mathrm{Gyr}$; however, in conjunction with a dynamo, such motions might be important in certain galaxies.

\subsection{Treatment of Galactic Turbulence}

Several basic gas components are involved in galactic turbulence. The disk consists of warm gas, interspersed by cold clouds and hot bubbles. Hot bubbles result from local heating (e.g. OB associations and supernova and superbubble explosions) and eject hot gas into the halo (galactic fountains). These violent motions, in addition to stellar winds, help to drive the turbulence. Furthermore, random motions of molecular clouds may stir up the warm gas, because they are dynamically coupled by magnetic field lines. The Parker instability may also be a source of turbulence, or it may at least act as an agent causing the movement of flux tubes and thereby generate an $\alpha$-effect (Parker 1992, Hanasz $\&$ Lesch 1993). In the model of Vázquez-Semadeni et al (1995), the turbulence is driven by gravity and density gradients that result from interstellar cooling and heating processes.

To understand the effect of these different gas components on the magnetic field we need to discuss the coupling of the magnetic field to those components. The magnetic fields in the hot component are rapidly ejected into the halo. They are then no longer directly important for magnetic processes in the disk, but are essential in the galactic halo. Clouds could be more important, because a large-scale field would be dragged with the gas into these clouds as they form, and the cloud motions would entangle the magnetic field lines (Beck 1991). This process is of only limited duration, because ambipolar diffusion (Mestel 1966) would decouple the clouds from the field on a timescale of $10^{7} \mathrm{yr}$. 
The outcome is that for most of the time the magnetic field remains attached to the diffuse ionized gas, and, to the extent that the field is associated with clouds, the effect of the clouds is to contribute to the turbulent dynamics of the magnetic field lines. Even if this is an important contributor to the chaotic driving of field lines (in addition to the turbulence mentioned above), it is reasonable to assume that the magnetic field in a galactic disk is on average linked to the warm, ionized medium and perhaps also to the warm neutral medium, both of which are in a turbulent state.

Dynamo action is well established from numerical turbulence simulations. In the absence of (rotational) velocity shear, the magnetic field is very intermittent (Meneguzzi et al 1981). In the presence of rotational shear, the resulting magnetic shear instability (e.g. Balbus \& Hawley 1992) can lead to strong large-scale fields (Brandenburg et al 1995a). This mechanism yields coherent fields similar to those in ordinary $\alpha \Omega$-dynamos.

The classical $\alpha$-effect quantifies the field-aligned electromotive force resulting from magnetic field lines twisted by the turbulence (cf simulations by Otmianowska-Mazur \& Urbanik 1994). In the original picture the dynamics of these field lines is governed by external turbulent motions. Parker (1992) discussed a new, perhaps more appropriate, concept in which the motions result mostly from the dynamics of magnetic field lines themselves. The concept of an $\alpha$-effect seems, however, sufficiently robust so that the form of the basic equations is always the same. In fact, the $\alpha$-effect is only one of many effects relating the mean emf $\mathcal{E} \equiv \overline{\mathbf{u} \times \mathbf{b}}$ to the mean magnetic field and its derivatives. If the mean field is not too intermittent, we can expand

$$
\mathcal{E}_{i}=\alpha_{i j} \bar{B}_{j}+\eta_{i j k} \frac{\partial \bar{B}_{j}}{\partial x_{k}}
$$

(Krause \& Rädler 1980), neglecting higher derivatives of $\overline{\mathbf{B}}$. This relation is used when solving the induction equation for the mean magnetic field,

$$
\frac{\partial \overline{\mathbf{B}}}{\partial t}=\nabla \times(\overline{\mathbf{u}} \times \overline{\mathbf{B}}+\mathcal{E}) .
$$

The mean velocity $\overline{\mathbf{u}}$ comprises both the rotational velocity, as well as galactic winds and any other large-scale flows. This is where the observed rotation curves and other large-scale flow components of individual galaxies enter into the theory and models.

The $\alpha_{i j}$ and $\eta_{i j k}$ tensors in (1) are anisotropic (Ferriére 1993, Kitchatinov et al 1994). Anisotropies can arise from stratification, rotation, shear, and magnetic fields. Stratification and rotation are most important, because without them there would be no $\alpha_{\phi \phi}$ component, which is needed to regenerate poloidal magnetic fields from $\bar{B}_{\phi}$. An important contribution to $\eta_{i j k}$ comes 
from isotropic turbulent magnetic diffusion, $\epsilon_{i j k} \eta_{\mathrm{t}}$, where $\eta_{\mathrm{t}}$ is the turbulent magnetic diffusivity. Explicit expressions in the framework of the first-order smoothing approximation (FOSA) were first derived by Steenbeck et al (1966) and Krause (1967), and more recently by Rüdiger \& Kitchatinov (1993). They find expressions of the form

$$
\alpha \approx-l^{2} \Omega \cdot \nabla \ln (\rho v) F(\overline{\mathbf{B}}, \Omega), \quad \eta_{\mathrm{t}} \approx \frac{1}{3} v l G(\overline{\mathbf{B}}, \Omega),
$$

where $l$ is the correlation length of the turbulence $\alpha$ stands for $\alpha_{\phi \phi}$, and $F$ and $G$ are certain ("quenching") functions. The stratification of $\rho v$ is important, because it breaks the symmetry between upward and downward motions. If $h$ is the scale height, a rough estimate gives

$$
|\alpha| \sim \min \left(\Omega l^{2} / h, v\right),
$$

which ensures that $\alpha$ does not exceed $v$ (e.g. Zeldovich et al 1983).

The FOSA is valid either for small magnetic Reynolds numbers (which is irrelevant here) or in the limit of short correlation times (which is also not well satisfied in the ISM). However, although higher order terms may become important, they affect the results only quantitatively (Zeldovich et al 1988, Carvalho 1992). There are independent attempts to compute the transport coefficients resulting from evolving flux tubes (Hanasz \& Lesch 1993) and from expanding supernovae and superbubbles rather than from turbulence (Ferriére 1993, Kaisig et al 1993). The resulting values of $\alpha$ and $\eta_{\mathrm{t}}$ are smaller than those expected from interstellar turbulence, suggesting that explosions are of lesser importance.

Turbulent diamagnetism (Zeldovich 1957) can be represented as a macroscopic velocity, $\overline{\mathbf{u}}_{\mathrm{dia}}=-1 / 2 \nabla \eta_{\mathrm{t}}$ (Roberts \& Soward 1975, Kitchatinov \& Rüdiger 1992). It tends to expel magnetic fields from regions where $\eta_{\mathrm{t}}$ is large. This term can be considered as a contribution to the antisymmetric part of $\alpha_{i j}$ (Rädler 1969). Additional effects of this kind are magnetic buoyancy (Moss et al 1990) and topological pumping (Section 7.2).

\subsection{Basic Galactic Dynamo Models}

The simplest form of the mean field $\left(\alpha^{2} \Omega\right)$ dynamo equation (2) that retains the basic physics (e.g. Parker 1979, Roberts \& Soward 1992) is, in dimensionless form,

$$
\frac{\partial \overline{\mathbf{B}}}{\partial t}=\nabla \times\left(R_{\omega} \overline{\mathbf{u}} \times \overline{\mathbf{B}}+R_{\alpha} F \alpha \overline{\mathbf{B}}-G \eta_{\mathrm{t}}, \nabla \times \overline{\mathbf{B}}\right),
$$

where $F(\overline{\mathbf{B}}, \Omega)=\left(1+\overline{\mathbf{B}}^{2} / B_{\text {eq }}^{2}\right)^{-1}$ is the simplest form of " $\alpha$-quenching" and $G(\overline{\mathbf{B}}, \Omega)=1$. Distances and times are measured in units of $h_{*}$ and $h_{*}^{2} / \eta_{\mathrm{t} *}$, 
respectively, where $\overline{\mathbf{u}}=\boldsymbol{\Omega} \times \mathbf{r}$ and $\alpha$ and $\eta_{\mathrm{t}}$ are normalized by appropriately chosen characteristic values denoted by asterisks. Dimensionless numbers

$$
R_{\omega}=h_{*}^{2} \Omega_{*} / \eta_{\mathrm{t} *}, \quad R_{\alpha}=h_{*} \alpha_{*} / \eta_{\mathrm{t} *}
$$

characterize the amplification of magnetic field by shearing of the mean velocity field and the $\alpha$-effect, respectively. Using Equation (3), $\alpha$ and $\eta_{\mathrm{t}}$ can be expressed through observable parameters of the galaxies such as the rotation curve, rms velocity and scale, and the thickness of the ionized disk (a function of $r$ ). The quenching effects also require that the gas density is specified as a function of position. Equation (5) must be supplemented by boundary conditions. In models that treat the disk alone, these are usually vacuum boundary conditions in which one assumes that the turbulent magnetic diffusivity outside the disk is infinite. This proves to be a reasonable approximation to reality (Moss \& Brandenburg 1992), as $\eta_{\mathrm{t}}$ varies by perhaps a factor of about 50 between the disk and the halo (see Brandenburg et al 1993, Poezd et al 1993). However, more advanced treatments employ the embedded disk model (Stepinski \& Levy 1988). This includes a spherical galactic halo and appropriate boundary conditions are imposed at the surface of the halo, whereas the disk is modeled by appropriate distributions of $\overline{\mathbf{u}}, \alpha$, and $\eta_{\mathrm{t}}$. This concept has proved sufficiently adaptable to accommodate developing requirements, such as the inclusion of a flared disk, an $\alpha$ effect extending into the halo (Section 7.1), and/or a galactic wind (Section 7.2).

Initial conditions for (5) are often chosen to correspond to a weak seed field. Exponentially growing solutions then arise, $\overline{\mathbf{B}} \propto \exp (\Gamma t)$, provided the dynamo number $D=R_{\alpha} R_{\omega}$ exceeds a certain value $D_{\text {crit }} \approx 10$. Using Equation (3) one can show that $D \simeq 9\left(h_{*} \Omega_{*} / v\right)^{2}$. For $h_{*} \simeq 500 \mathrm{pc}, \Omega_{*} \simeq 20 \mathrm{~km} \mathrm{~s}^{-1} \mathrm{kpc}^{-1}$, and $v \simeq 10 \mathrm{~km} \mathrm{~s}^{-1}$ we obtain $D \simeq 10$, so that the dynamo is expected to operate under typical galactic conditions. For $D \gg D_{\text {crit }}$, the growth rate is estimated as $\Gamma \simeq C D^{1 / 2} \eta_{\mathrm{t}} / h_{*}^{2} \simeq C\left(\alpha_{*} \Omega_{*} h_{*}\right)^{1 / 2}$, with $C$ a quantity of order unity depending on the galaxy model. A typical model gives $\Gamma^{-1} \simeq 5 \times 10^{8} \mathrm{yr}$; this is a lower estimate for the dynamo timescale. [We note, however, that the timescale for the magnetic shear instability is the inverse Oort $A$-value (Balbus \& Hawley 1992), which is somewhat shorter $\left(10^{8} \mathrm{yr}\right)$. This mechanism leads to dynamo action (Brandenburg et al 1995b) that would lower the effective value of $\Gamma^{-1}$.]

All classical dynamo models predict that the large-scale field in the outer parts of the disks in spiral galaxies has quadrupole (S0) symmetry, that is, both $\bar{B}_{r}$ and $\bar{B}_{\phi}$ are even in $z$, whereas $\bar{B}_{z}$ is odd (Parker 1971, Vainshtein \& Ruzmaikin 1971). This mode is dominant in a disk (but not in a sphere). A dipole (A0) mode, with both $\bar{B}_{r}$ and $\bar{B}_{\phi}$ odd in $z$ and $\bar{B}_{z}$ even, can be dominant near the axis of the disk. The large-scale field is amplified until $\alpha$ becomes significantly quenched, which occurs when $\bar{B}$ is of order $B_{\text {eq }}$, typically a few $\mu \mathrm{G}$. 

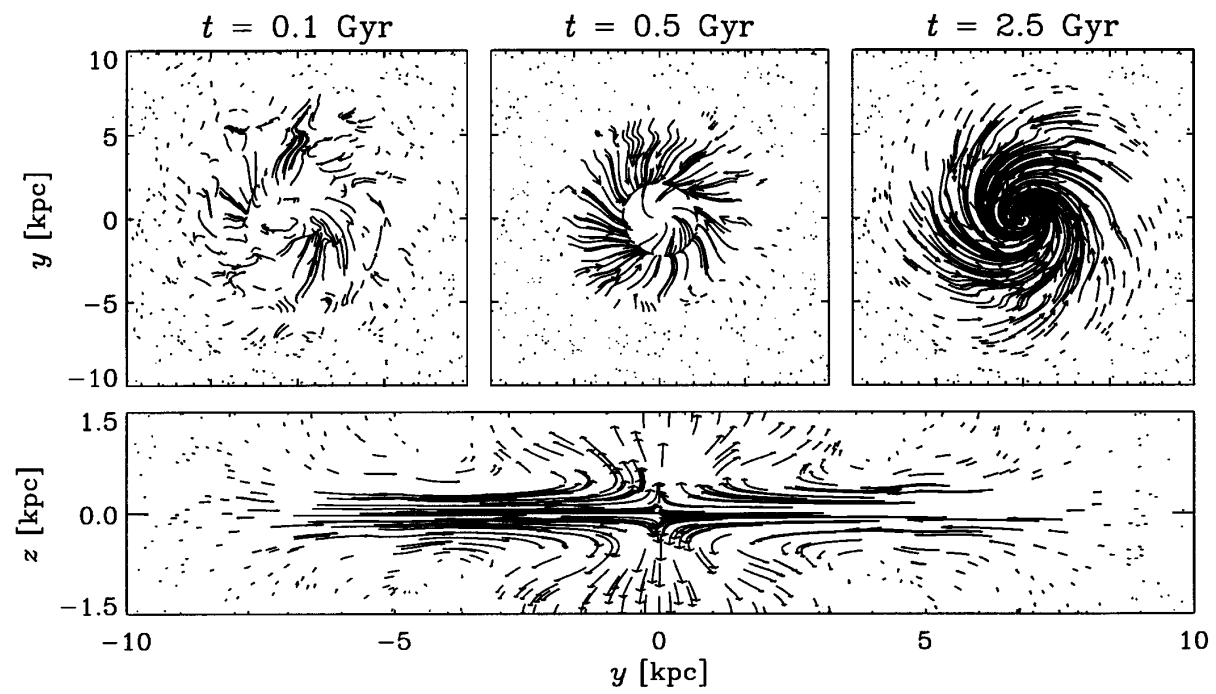

Figure 7 Face-on views showing the evolution of the magnetic field in a model of M83 (from KJ Donner \& A Brandenburg, in preparation). The lower panel gives an edge-on view for $t=8.1$ Gyr.

Field evolution is qualitatively different if the initial field is a random field with strength close to $B_{\text {eq }}$. There is then no kinematic stage, because $\alpha$ quenching is immediately important. The dynamo acts then to change the scale and spatial distribution of the field. An example of typical evolution of the magnetic field in a spiral galaxy as envisaged by the standard dynamo model is illustrated in Figure 7.

Over the past 5 to 10 years a large number of galactic dynamo models have been developed. The minimum ingredient of such models is a flat geometry. Such models were first computed in the 1970s, but computers can only now reach the regime applicable to the theory of asymptotically thin disks (Walker \& Barenghi 1994 and references therein). Galactic models share the somewhat frustrating property that nonaxisymmetric solutions are always harder to excite than axisymmetric ones (Ruzmaikin et al 1988a, Brandenburg et al 1990, Moss $\&$ Brandenburg 1992). Not even the inclusion of anisotropies seems to change this conclusion (Meinel et al 1990). Stable nonaxisymmetric solutions have only been found if $\alpha$ and $\eta_{\mathrm{t}}$ vary azimuthally (Moss et al 1991, 1993a; Panesar $\&$ Nelson 1992). The inclusion of nonlinear effects demonstrated that mixed parity states can persist over rather long times, even comparable with galactic lifetimes (Moss \& Tuominen 1990, Moss et al 1993a). When $\eta$-quenching is 
included $(G \neq 1)$, linear calculations show that $\mathrm{A} 0$ and $\mathrm{S} 1$ modes may be more readily excited (Elstner et al 1996).

In most of these models $\alpha_{i j}$ and $\eta_{i j k}$ were adopted using qualitative forms of (3) and (4), calibrated by observations. Significant conceptual progress has been made recently by deriving all these functions consistently from the same turbulence model, which includes stratification of density and turbulent velocity, derived from a condition of hydrostatic equilibrium (Schultz et al 1994, Elstner et al 1996). One should not forget, however, that such models still rely on important approximations and simplifications (e.g. FOSA and the lack of a reliable turbulence model).

\subsection{The Quenching Problem}

In recent years the feedback of the magnetic field on the turbulent diffusion and the $\alpha$-effect has become a topic of major concern. Piddington (1970) was the first to suggest that for large magnetic Reynolds numbers the magnetic fluctuations would be strong enough to suppress turbulent diffusion. This idea was rejected by Parker (1973), who argued that the development of strong smallscale fields is limited by reconnection, so that they do not hinder turbulent mixing of field and fluid. In fact, without turbulent diffusion the galactic differential rotation would wind up the field so tightly that it would not resemble the magnetic field structure of any observed galaxy (Section 8.3).

The results of the two-dimensional numerical MHD experiment of Cattaneo \& Vainshtein (1991) stimulated new interest in the problem of turbulent diffusion. They found that $\eta_{\mathrm{t}}$ is suppressed according to $\eta_{\mathrm{t}}=v l /\left(1+R_{\mathrm{m}} \overline{\mathbf{B}}^{2} / B_{\mathrm{eq}}^{2}\right)$, where $R_{\mathrm{m}}=v L / \eta$ is the magnetic Reynolds number based on the microscopic diffusivity. Evidently, $\eta_{\mathrm{t}}$ would be significantly reduced when $\bar{B}$ is comparable to $R_{\mathrm{m}}^{-1 / 2} B_{\text {eq }}$. In galaxies, $R_{\mathrm{m}}=\mathcal{O}\left(10^{17}\right)$, so $\eta_{\mathrm{t}}$ would essentially be zero. Even if we used a Reynolds number based on ambipolar diffusion, with $R_{\mathrm{m}}^{\mathrm{AD}} \gtrsim O\left(10^{3}\right), \eta_{\mathrm{t}}$ would still be too small. This type of quenching is much stronger than the "traditional" quenching (Moffatt 1972), so something seems to be wrong (e.g. Field 1996).

In three dimensions the turbulent motions would continue to entangle the magnetic field in the direction perpendicular to $\overline{\mathbf{B}}$ (Krause \& Rüdiger 1975, Parker 1992). This has now also been demonstrated numerically (Nordlund et al 1994) as well as analytically (Gruzinov \& Diamond 1994). In other words, turbulent diffusion is really not significantly suppressed at field strengths somewhat below the equipartition value. The decay of sunspots is a good example of this (Krause \& Rüdiger 1975).

Vainshtein \& Cattaneo (1992) and Tao et al (1993) suggested that the $\alpha$-effect might also be quenched dramatically, $\alpha=\alpha_{\text {kin }} /\left(1+R_{\mathrm{m}} \overline{\mathbf{B}}^{2} / B_{\text {eq }}^{2}\right)$, where $\alpha_{\text {kin }}$ 
is the kinematic value of Equation (3). The analysis of Gruzinov \& Diamond (1994) seems to support this result. On the other hand, the simulations of Tao et al (1993), as well as unpublished simulations by A Brandenburg, are reminiscent of an earlier result by Moffatt (1979), that the $\alpha$-effect may fluctuate strongly and never converge to a finite value if $R_{\mathrm{m}}$ is large.

There is at present no conclusive resolution to this problem, but here are some possibilities: ( $a$ ) The conventional $\alpha$-effect might still work in reality, but the method used to estimate $\alpha$ from simulations is inappropriate (e.g. the boundary conditions preserve the magnetic flux, so the $\alpha$-effect is forced to have zero effect on the average field; or the computational domain might be too small compared to the eddy size). (b) The conventional $\alpha$-effect is really nonexistent, but instead some other mechanism (e.g. an inverse cascade mechanism, incoherent $\alpha$-effect, or cross-helicity effect) generates large-scale fields in conjunction with shear. (c) An important contribution to $\alpha$ comes from the Parker instability: This mechanism would work especially for finite magnetic fields.

A somewhat different problem was raised by Kulsrud \& Anderson (1992), who suggested that the growth of large-scale fields is suppressed by ambipolar diffusion at small scales. However, before we can draw any final conclusions, nonlinear effects need to be included. These can be important for two reasons: The inverse cascade process is inherently nonlinear, and nonlinear ambipolar diffusion can lead to sharp magnetic structures (Brandenburg \& Zweibel 1995), which would facilitate fast reconnection and rapidly remove magnetic energy at small scales.

The problem raised by Vainshtein \& Cattaneo (1992) is related to the assumption that most of the magnetic energy is at small scales, i.e. $\left\langle\mathbf{B}^{2} \gg\right\rangle \mathbf{B}^{2}$. This, however, is only a result of linear theory and is not supported by observations (Section 3). A recent simulation by Brandenburg et al (1995a) is relevant in this context. Here a large-scale field is generated with $\left\langle\mathbf{B}^{2}\right\rangle /\left\langle\mathbf{B}^{2}\right\rangle \approx 0.5 \gg R_{m}^{-1 / 2} \approx$ 0.1 . The dynamo works even in the presence of ambipolar diffusion, which Kulsrud \& Anderson (1992) thought to be effective in destroying large-scale dynamo action. Here, the incoherent $\alpha$-effect is much larger than the coherent effect, but the estimated value of the dynamo number is nevertheless above the critical value, suggesting that conventional dynamo action might also be at work.

\section{ORIGIN OF GALACTIC MAGNETIC FIELDS}

\subsection{Cosmological Magnetic Fields}

Zeldovich (1965) noted that a Friedmannian cosmology admits a weak uniform magnetic field given as an initial condition at the Big Bang (see also Zeldovich \& Novikov 1982, LeBlanc et al 1995). A hypothetical homogeneous magnetic 
field in the Universe has been never detected and only its upper limits are available. A uniform magnetic field $\bar{B} \gtrsim 10^{-7} \mathrm{G}$ at the present day would lead to anisotropy in the expansion of the Universe, thereby affecting nucleosynthesis (e.g. Cheng et al 1994, Grasso \& Rubinstein 1995). Analysis of Faraday rotation measures of extragalactic sources gives a stronger upper limit of $10^{-9}$ $10^{-10} \mathrm{G}$ (Ruzmaikin \& Sokoloff 1977). A magnetic field leads to transitions between left- and right-handed neutrinos (spin-flip) in the early Universe. Nucleosynthesis gives an upper limit to the abundance of right-handed neutrinos and thus yields the constraint

$$
\bar{B}_{\text {proto }} \lesssim(1-30) \times 10^{-13} \mathrm{G}
$$

for the present-day uniform cosmological field (Sciama 1994).

Taking a cosmological magnetic field as a given initial condition at the Big Bang is rather unsatisfactory. Furthermore, it is not clear whether a homogeneous magnetic field can be incorporated into modern quantum cosmology, where it cannot be prescribed as an initial condition.

Several mechanisms of small-scale magnetic field generation by quantum effects in the early Universe have been proposed (Turner \& Widrow 1988, Quashnock et al 1989, Vachaspati 1991, Ratra 1992). The resulting spatial scales of cosmological magnetic fields are very small and, even after cosmological expansion, they are negligible in comparison with protogalactic scales.

The strength and scale of the relic magnetic field can be estimated as follows. As magnetic diffusion smoothes the field, its scale at time $t$ will be about $(\eta t)^{1 / 2}$, where $\eta$ is the magnetic diffusivity, as the initial scale is much smaller. At the epoch of nucleosynthesis, the resulting scale is $10^{4} \mathrm{~cm}$, corresponding to a scale $l \approx 10^{-6} \mathrm{pc}$ today. The same arguments as for Equation (7) give an upper limit on the magnetic field at nucleosynthesis of $10^{11} \mathrm{G}$. With allowance for a change in the equation of state at $t=t_{*} \approx 10^{4} \mathrm{yr}$, the frozen-in magnetic field at time $t$ is diluted by cosmological expansion to $b\left(t_{*} / t\right)^{4 / 3}\left(1 \mathrm{~min} / t_{*}\right)^{1 / 3}$. Since the protogalaxy includes $(L / l)^{3}$ correlation cells, the average field strength is smaller by a factor $(L / l)^{-3 / 2}$. This yields the following upper limit on the average magnetic field at the scale of the protogalaxy at the present time,

$$
\bar{B}_{\text {proto }} \lesssim 2 \times 10^{-23} \mathrm{G}
$$

(see Enqvist et al 1993, 1995). Thus either the cosmological magnetic field is exactly homogeneous, and then the restriction (7) applies, or the field was produced in the early Universe, and then it must satisfy (8). We should note that the above estimates neglect ohmic losses. These constraints do not apply to magnetic fields generated at later stages of cosmological evolution. Battery 
mechanisms can contribute at more recent epochs, giving (Mishustin \& Ruzmaikin 1971, see also Harrison 1970, Baierlein 1978),

$$
\bar{B}_{\text {proto }} \lesssim 10^{-21} \mathrm{G} \text {. }
$$

\subsection{The Primordial Origin of Galactic Magnetic Fields}

We now assess the possibility that the large-scale magnetic field observed in galaxies is merely a result of the twisting of a cosmological magnetic field by galactic differential rotation (see e.g. Kulsrud 1986). Aiming at conservative estimates, we neglect any magnetic field dissipation. An isotropic contraction of the protogalaxy with a frozen-in magnetic field, from an intergalactic density $\rho_{\mathrm{IG}} \approx 10^{-29} \mathrm{~g} \mathrm{~cm}^{-3}$ up to an interstellar density $\rho \approx 10^{-24} \mathrm{~g} \mathrm{~cm}^{-3}$, results in amplification of the primordial magnetic field by a factor of $2 \times 10^{3}$. Differential rotation results in an amplification of the magnetic field in a young galaxy by the number of galactic rotations in $10^{10} \mathrm{yr}$, which is $N \sim 30$. Altogether, a conservative upper limit on the field in the galactic disk resulting from a primordial field is

$$
\bar{B}_{\text {proto }} N\left(\rho / \rho_{\mathrm{IG}}\right)^{2 / 3}<2 \times 10^{-7} \mathrm{G},
$$

where the more favorable constraint (7) has been used. A primordial field wound up by differential rotation ultimately decays: In a region with closed streamlines (a galaxy in this case) this effect is known as flux expulsion (Moffatt 1978).

\subsection{The Dynamo Origin of Magnetic Field}

Any dynamo requires a seed field because Equation (5) is homogeneous in $\bar{B}$. There are two possibilities for the seed field: It can be essentially of cosmological origin or it can result from processes occurring in the ISM.

The large-scale dynamo timescale in a typical galaxy cannot be shorter than $\tau \sim 5 \times 10^{8} \mathrm{yr}$ (see Section 4.4). A primordial field on a protogalactic scale then needs to be at least $O\left(10^{-18}\right) \mathrm{G}$ in order to be amplified to $10^{-6} \mathrm{G}$ in $10^{10}$ $\mathrm{yr}$ (when the amplification by protogalaxy contraction is considered). With the estimates (8) and (9), we conclude that a cosmological magnetic field is not viable as a seed field for a galactic dynamo. Moreover, for the Milky Way and M31, the timescale is more like $\tau \approx 10^{9} \mathrm{yr}$, so that for these galaxies a primordial magnetic field needs to be at least about $2 \times 10^{-14} \mathrm{G}$, assuming that $\tau$ has not varied significantly during galactic evolution.

A sufficiently strong seed field for the large-scale galactic dynamo can be generated by a small-scale dynamo. The scale height of the disk of a young galaxy is estimated as $h \simeq 100-500$ pc (Briggs et al 1989) and the turbulent 
velocity as $v \simeq 10 \mathrm{~km} \mathrm{~s}^{-1}$ (Turnshek et al 1989). Assuming that $l \simeq 100$ $300 \mathrm{pc}$ and $\rho \simeq 10^{-24} \mathrm{~g} \mathrm{~cm}^{-3}$, we conclude that a random magnetic field $b \simeq$ $\left(4 \pi \rho v^{2}\right)^{1 / 2} \simeq 2-2.5 \mu G$ of a scale $100-300 \mathrm{pc}$ is generated by the fluctuation dynamo on a timescale of order $\tau_{1} \sim l / v \simeq 10^{6}-10^{7}$ yr. Because a galactic disk contains about $N_{1}=(h / l)(R / l)^{2}$ turbulent cells, the resulting mean field dynamo seed field is about $b N_{1}^{-1 / 2} \simeq 10^{-8} \mathrm{G}$. This is much larger than possible cosmological seed fields $(8,9)$, even if the field compression during galaxy formation is taken into account.

The resulting small-scale field is strong enough to produce, via a mean field galactic dynamo, a large-scale magnetic field of $\mu \mathrm{G}$-strength in $\sim(1-2) \times 10^{9}$ $\mathrm{yr}$ (Beck et al 1994a). This means that even the presence of regular magnetic fields in galaxies with redshifts of $z \simeq 2$ or even $z \simeq 3.4$ (Wolfe et al 1992, White et al 1993) does not contradict the picture of generation and maintenance of large-scale fields by a mean-field dynamo mechanism. The possible role of the halo (Chiba \& Lesch 1994) and radial motions (Camenzind \& Lesch 1994) has also been investigated.

The fluctuation dynamo also needs a seed but, because of the very short fluctuation dynamo timescale, even the magnetic fields generated by the battery effects in stars (Biermann 1950, Mestel \& Roxburgh 1962), and subsequently ejected into the ISM, or a cosmological field (Section 5.1) would suffice.

Thus, large-scale dynamo action in a galaxy is preceded by a small-scale dynamo that prepares the seed for the former. These may operate at different epochs. Small-scale dynamo action has been considered by Pudritz \& Silk (1989) for the protogalaxy, by Zweibel (1988) during the post-recombination epoch, and before recombination by Tajima et al (1992).

A rather radical view of the role of the Galactic center in the origin of the global galactic magnetic field was proposed by Hoyle (1969), who suggested that the magnetic field observed in the solar vicinity had been ejected from the Galactic center. This idea was rejected because the required magnetic field in the nucleus is $10^{9} \mathrm{G}$, and its energy exceeds the gravitational energy of a black hole with a mass of $10^{8} M_{\odot}$. Nevertheless, Chakrabarti et al (1994) proposed a similar hypothesis, with the azimuthal field being amplified up to $\bar{B}_{\text {core }} \simeq 3 \times 10^{5}$ $\mathrm{G}$ within $r_{0} \simeq 3 \times 10^{11} \mathrm{~cm}$ of the center. A galactic wind is then supposed to carry this field to the outer parts of the Galaxy. However, this gives for the solar vicinity an extremely weak field of $\bar{B} \simeq\left(r_{0} / r_{\odot}\right)\left(h_{0} / h_{\odot}\right) \bar{B}_{\text {core }} \simeq$ $6 \times 10^{-16} \mathrm{G}$, where $h_{0} \simeq r_{0}$ and $r_{\odot}=8.5 \mathrm{kpc}$ and $h_{\odot}=500 \mathrm{pc}$ are the radius and half-thickness of the magnetoionic disk in the Solar vicinity. Chakrabarti et al obtained for $\bar{B}$ a value about $10^{10}$ times larger by overlooking a factor $h_{0} / h_{\odot}$. 


\section{EFFECTS OF THE DYNAMO ENVIRONMENT}

\subsection{Starbursts}

Starburst galaxies are believed to contain regions of strongly enhanced star formation, particularly of massive stars. The rapid evolution of these stars, through phases with energetic stellar winds to supernovae, may possibly make the turbulence more energetic (for example, by increasing the fraction of hot gas and hence the mean sound speed), with several possible consequences for dynamo theory. Any increased turbulent pressure will inflate the disk, and the $\alpha$ effect may be enhanced above the value appropriate to a quiescent galaxy. Both of these effects increase the dynamo number (Section 4.4). This enhancement may be preferentially concentrated in azimuth, perhaps lagging the spiral arms. Ko \& Parker (1989) suggested that galactic dynamos may turn on and off in response to changing starburst activity. However, the timescale for starbursts is believed to be less than $10^{8} \mathrm{yr}$, which is certainly no longer (and possibly considerably shorter) than a dynamo growth time. Thus it is hard to see how significant field growth can be caused by isolated starburst episodes; see also Vallée (1994). Nozakura (1993) presented a local model with several feedback loops, linking star formation via gravitational instability, dynamo action, and energy release into the ISM via supernovae. In some contrast to Ko \& Parker, he concluded that there was only a limited parameter range in which strong star formation and dynamo action could coexist: Essentially star formation requires a high surface density of gas and/or a low sound speed, and so a thin disk, giving a smaller dynamo number. These are clearly matters requiring further attention. Moreover, in an active galaxy, fountain flows will be more frequent, enhancing the lifting of field from the disk into the halo-see Section 7.2.

\subsection{Galactic Encounters}

There is strong observational evidence that a number of spiral galaxies are interacting gravitationally with a neighbor. The clearest nearby example is M81, which is believed to have undergone a recent encounter with NGC 3077 (probably less than $10^{9} \mathrm{yr}$ ago). Because the orbit of NGC 3077 is approximately in the disk plane of M81, this system is particularly well suited to simulation, and Thomasson \& Donner (1993) predict nonaxisymmetric velocities of order 10 $\mathrm{km} \mathrm{s}^{-1}$ in the disk of M81. With $\eta_{\mathrm{t}} \sim 10^{26} \mathrm{~cm}^{2} \mathrm{~s}^{-1}$ and $L \sim 1 \mathrm{kpc}$, the magnetic Reynolds number $U L / \eta_{\mathrm{t}}$ is then about 30, quite large enough to affect significantly the disk fields (Vallée 1986). Interestingly, M81 appears to have a strong bisymmetric field component. M33 also may have some bisymmetric field structure, and it is believed to be interacting with M31. Recently, at least weak evidence has been found for BSS in the interacting galaxy NGC 2276 (Hummel $\&$ Beck 1995) and for MSS in M51 (EM Berkhuijsen et al, in preparation). 
If we consider a Fourier decomposition of $\overline{\mathbf{u}}$ and $\overline{\mathbf{B}}$ into parts $\overline{\mathbf{u}}_{m}, \overline{\mathbf{B}}_{m}$, corresponding to an azimuthal wave number $m$, then the induction term $\nabla \times(\overline{\mathbf{u}} \times \overline{\mathbf{B}})$ can give rise to a bisymmetric field component in two ways. If the dynamo basically generates an axisymmetric field $\overline{\mathbf{B}}_{0}$, then $\overline{\mathbf{u}}_{1}$ can generate a slaved $m=1$ component $\overline{\mathbf{B}}_{1}$ from the $\overline{\mathbf{u}}_{1} \times \overline{\mathbf{B}}_{0}$ interaction. Moss et al (1993b) investigated this possibility in a nonlinear model with a relatively thick disk, using a velocity field based on the Thomasson \& Donner (1993) simulation. They found that a globally modest bisymmetric field component could be generated, concentrated to the outer part of the disk, where it may dominate. More subtly, the $\overline{\mathbf{u}}_{2} \times \overline{\mathbf{B}}_{1}$ interaction (giving rise directly to $m=1$ and $m=3$ field components) may be such as to increase the linear growth rate of the bisymmetric field component compared to that of the axisymmetric component, so that in the nonlinear case a substantial bisymmetric field could survive. Moss (1995) showed that, in a simple linear model, the $m=1$ field could then be excited at lower dynamo number than the $m=0$ field, but a nonlinear investigation using a more realistic model is needed to clarify the importance of this mechanism. The remarks concerning the modal interactions apply, of course, whatever the mechanism providing the velocity field. In particular, it may be relevant that a $\overline{\mathbf{u}}_{2} \times \overline{\mathbf{B}}_{0}$ interaction can give rise to a slaved $m=2$ field component.

\subsection{Parametric Resonance with Spiral Arms}

A dynamo mechanism with selective amplification of BSS caused by swing excitation by the spiral arms has been proposed by Chiba \& Tosa (1990). Unlike axisymmetric dynamo modes (which do not oscillate at realistic dynamo numbers), a bisymmetric magnetic field has the form of a dynamo wave, which propagates in the azimuthal direction as seen in an inertial frame. Because the spiral pattern modulates the dynamo efficiency, a parametric resonance between the spiral arms and the bisymmetric magnetic field might be expected. Applying the classical theory based on the Mathieu equation (see Landau \& Lifshitz 1969), Chiba \& Tosa argued that the $m=1$ mode is amplified when its frequency $\omega_{B}$ is half that of the spiral pattern, $\omega_{\mathrm{SP}}$, and the growth rate of the $m=1$ mode is increased proportionally to the increment of the dynamo number in a spiral arm. However, the classical theory of parametric resonance is valid only for simple, discrete, stable oscillatory systems and may not apply to a dynamo system (Schmitt \& Rüdiger 1992).

Parametric resonance in a galactic dynamo, which is a distributed oscillatory system, was considered asymptotically in the thin-disk approximation by Kuzanyan \& Sokoloff (1993). They showed that the resonant condition remains the same in terms of frequencies, but the resulting enhancement in the growth rate is much smaller than above and is proportional to the efficiency 
of the radial diffusive transport of the magnetic field, i.e. the aspect ratio of the disk $h / R$. Galactic parametric resonance has also been investigated numerically for a thin-disk model, keeping two explicit space directions, $r$ and $\phi$ (Moss 1996). These results confirm that the effect is weaker than for a classical parametric resonance and, furthermore, demonstrate that the resonance remains efficient for a larger mismatch between $2 \omega_{B}$ and $\omega_{\mathrm{SP}}$ than implied by the Mathieu equation. Since the equality $2 \omega_{B}=\omega_{\mathrm{SP}}$ is not an intrinsic property of galaxies, this finding is very helpful for practical applications. Nevertheless, parametric resonance can be expected to occur at most in a fraction of galaxies, where these quasi-independent frequencies satisfy the appropriate condition.

Other attempts to enhance the effect involve dynamo solutions that oscillate even in the lowest approximation in $h / R$ (Hanasz et al 1991, Hanasz \& Chiba 1993), i.e. in the local dynamo equation. Such oscillatory solutions arise only for unrealistically large dynamo numbers, requiring a downward revision of the turbulent magnetic diffusivity by a factor of 10 (Hanasz \& Lesch 1993).

A further type of parametric resonance that can occur only in a distributed system such as a galactic dynamo has been suggested by Mestel \& Subramanian (1991) and Subramanian \& Mestel (1993). They assume that the dynamo wave is comoving with a spiral arm and that the dynamo efficiency is larger inside the arm than in the interarm space. The resulting growth rate of the magnetic field, captured by the arms, is larger than on average over the disk; the resonance condition is thus $\omega_{B}=\omega_{\mathrm{SP}}$. The resulting (regular) magnetic field is connected with the spiral arms rather than with the disk as a whole; in particular, significant vertical magnetic fields might be expected. It is not completely clear whether or not this mechanism favors the bisymmetric mode over the axisymmetric one. The predictions of these models deserve a careful confrontation with observations.

\subsection{Contrast Structures}

Suppose that the seed magnetic field in one part $G_{1}$ of a thin galactic disk has approximately the form of a growing eigensolution, while in another part $G_{2}$ the seed magnetic configuration is close to the same eigensolution, but with the opposite sign. After some time, advection and diffusion will bring these regions of oppositely directed magnetic fields into contact. The neutral surface at the boundary of these regions will move due to diffusion and advection, so the final stage of magnetic field evolution will be determined by magnetic field propagated, say, from the part $G_{1}$. The motion of the neutral magnetic surface is governed by the competition between advection and diffusion of field from $G_{1}$ towards $G_{2}$ and vice versa. Provided the nonlinear stage of magnetic field evolution begins before the field attains the form of the leading 
eigensolution, these two can balance each other. This balance is possible only if the neutral surface is at some special location in the galactic disk; then a long-lived magnetic structure appears (Belyanin et al 1994). This type of nonlinear solution of the dynamo equations is known as a contrast structure. The thickness of the transition region between $G_{1}$ and $G_{2}$ is approximately the disk thickness, and its lifetime can even be as long as the diffusion time along the disk, $R^{2} / \eta_{\mathrm{t}} \sim 10^{11} \mathrm{yr}$. Inside the contrast structure, annihilation of the oppositely directed magnetic fields is balanced by generation and advection, similar to a solitonís behavior in the nonlinear wave equation.

Contrast structures in purely axisymmetric disks are expected to be most often axisymmetric, because they are not affected by differential rotation. In the Milky Way, such axisymmetric contrasting structures can survive until today, and they may be identified with the reversals discussed in Section 3.8.2 (Poezd et al 1993). Contrast structures supported by nonaxisymmetric velocity and density distributions might explain the dominance of BSS in some galaxies (Moss et al 1993b; D Moss, in preparation; A Bikov et al, in preparation).

\subsection{The Influence of Magnetic Fields on the Galactic Disk}

Early ideas that magnetic fields might universally give rise directly to spiral structure have now generally been abandoned, because large-scale fields would need to have strengths $\gtrsim 10 \mu \mathrm{G}$ to cause the velocity perturbations of about 20 $\mathrm{km} \mathrm{s}^{-1}$ associated with spiral arms (e.g. Binney \& Tremaine 1987, p. 394). This can be compared with typical values of a few $\mu \mathrm{G}$ (Section 3.9). (Note that the above estimate is valid for a gas density appropriate to the Milky Way, and that for gas-rich galaxies, which tend to have larger fields, it would also be increased.) However, Nelson (1988) suggested, from study of a simplified, two-dimensional model, that magnetic fields might have a significant effect on gas dynamics at large galactocentric distances, where the gas density is lower.

Nevertheless, there may be more subtle effects. Magnetic pressure contributes significantly to the overall pressure balance in the ISM (e.g. Bowyer et al 1995), perhaps affecting the vertical distribution of the gas [scale height, etc (see Boulares \& Cox 1990)]. This in turn can affect the dynamo efficiency, establishing a feedback loop (Dobler et al 1996). Magnetic fields, of both large and small scale, could affect the formation and motion of clouds, for example, by increasing their effective cross-section. More directly, magnetic fields are believed to mediate the star-formation process, inter alia helping to solve the "angular momentum problem" (see Mestel 1985). A locally stronger magnetic field may bias the initial mass function to more massive stars (e.g. Mestel 1989), which, with their more rapid and violent evolution, could result in a more energetic ISM and perhaps an enhanced $\alpha$-effect, thus providing another feedback loop (Mestel \& Subramanian 1991, see also the discussion by Nozakura 1993). 
Even the relatively modest azimuthal magnetic torques might affect the centrifugal balance sufficiently to give a significant angular momentum transport. An investigation by Rüdiger et al (1993) suggests that in the case of fields of quadrupolar parity, a substantially subsonic gas inflow will result, with only a small effect on the dynamo field structure.

\section{MAGNETIC FIELDS IN HALOS}

From observations of external galaxies, magnetic fields are inferred in halos of spiral galaxies to distances of at least $5 \mathrm{kpc}$ and maybe even $10 \mathrm{kpc}$ from the disk plane, significantly beyond a synchrotron scale height (cf Section 3.6). Recently, dynamo models have directed some attention to out-of-disk fields. Here we address the two logical possibilities (while noting that they are not mutually exclusive): that such fields are generated in situ in the halo or that they are generated in the disk and then transported into the halo.

\subsection{In Situ Generation}

Interpretations of observations in the Milky Way suggest the presence of turbulent velocities of at least $50 \mathrm{~km} \mathrm{~s}^{-1}$ in galactic halos, compared to estimates of $10 \mathrm{~km} \mathrm{~s}^{-1}$ in disks. If we assume a length scale of order $0.5 \mathrm{kpc}$ and that halo angular velocities are comparable with those in the disk, we get canonical estimates of $\alpha \sim 3 \mathrm{~km} \mathrm{~s}^{-1}$ and $\eta_{\mathrm{t}} \sim 5 \times 10^{27} \mathrm{~cm}^{2} \mathrm{~s}^{-1}$, to be compared with $\eta_{\mathrm{t}} \sim 10^{26} \mathrm{~cm}^{2} \mathrm{~s}^{-1}$ in the disk. [See, e.g. the discussion in Poezd et al (1993). Note that Schultz et al (1994) adopt halo turbulent velocities that are much smaller than those in the disk: This may be a direct consequence of their turbulence model with $\alpha \propto \partial\left\langle v^{2}\right\rangle / \partial z$.] Taking $L \sim 10 \mathrm{kpc}$ gives standard dynamo numbers $R_{\alpha}=\alpha L / \eta_{\mathrm{t}} \sim 2$ and $R_{\omega}=\Omega_{0} L^{2} / \eta_{\mathrm{t}} \sim 200$. These are large enough for a dynamo to be excited (Ruzmaikin et al 1988a, Section VIII.1; Kahn \& Brett 1993). Note that such a dynamo would operate in a quasi-spherical volume, rather than a thin disk, that standard spherical $\alpha \Omega$ dynamos preferentially excite fields of dipolar (A0) topology, and that these are then often the only stable solutions of the full nonlinear equations. In contrast, S0 fields are usually preferred in thin disks. This situation immediately suggests the interesting possibility of simultaneous excitation of dynamo fields of opposite parity types in the two subsystems (halo and disk) (see Sokoloff \& Shukurov 1990). A priori, the possible existence of magnetic structures asymmetric with respect to the midplane, of neutral sheets, and of other nonstandard phenomena cannot be dismissed, as has been shown in some detail by Brandenburg et al (1992). Growth times in the halo are substantially longer than in the disk, and the halo field may still be in a transient state after a Hubble time. Detailed integrations show that, starting from a seed field of mixed parity, the overall field is initially 
dominated by S0 topology and concentrated in the disk. This phase can persist for order a Hubble time, but the final configuration is usually of A0 type, and may even be oscillatory. Given the long-lived transient phase with mixed parity fields present, observers today may be presented not with the eventual stable configuration, but rather an intermediate state of quite arbitrary geometry. Note that magnetic fields in the disk and halo of M51 are oppositely directed (EM Berkhuijsen et al, in preparation): This argues for in situ generation. More satisfactory halo models will need better data than is currently available on the dependence of the angular velocity in the halo on $z$, but these results seem qualitatively robust. To summarize, in some circumstances, dynamo theory may not be able to make detailed predictions about field geometries in specific galaxies.

A largely unexplored possibility is that some sort of Ponomarenko ("screw") dynamo (e.g. Ruzmaikin et al 1988c) might operate in the halo, if large-scale quasi-radial outflows ("winds") are twisted into helical form by the galactic rotation. Such dynamos excite nonaxisymmetric fields. If we take a simple model investigated by Ruzmaikin et al and use their definitions, then a wind velocity of $100 \mathrm{~km} \mathrm{~s}^{-1}$ and a typical galactic angular velocity gives a magnetic Reynolds number $R_{\mathrm{M}}$ large enough for the dynamo to work. Naively, the minimum $e$-folding time would be about $10^{9} \mathrm{yr}$, but this increases as $R_{\mathrm{M}}^{1 / 2}$ for larger $R_{\mathrm{M}}$, because the screw dynamo is "slow." These estimates suggest that the mechanism might be of marginal importance in halos, but real galaxy velocity fields are likely to be less efficient dynamos than the idealized forms considered by Ruzmaikin et al. We note in passing that Spencer \& Cram (1992) have discussed models of field amplification in which meridional flows ("winds") appear to play a central role. However, they solve the problem purely in the disk region; moreover, their solutions do not represent dynamo generation but rather local compression of field and hence the relevance to field generation processes in galaxies is unclear.

\subsection{Transport Out of the Disk}

Evidence for the existence of galactic winds, with speeds $U$ of hundreds of kilometers per second, is seen in some galactic halos, notably NGC 4631 and M82 (Section 3.6), implying turbulent magnetic Reynolds numbers $R_{\mathrm{M}}=$ $U L / \eta_{\mathrm{t}}$ of order 100. Strong field freezing will thus occur and, since the wind advection time $(L / U)$ is much shorter than the dynamo growth time, the wind will markedly affect the near-disk fields. For halo magnetic fields that are strong enough for their energy density to be comparable with the kinetic energy density of the wind, the dynamical effect of the field on the wind needs also to be considered, as in the analogous stellar wind problem, although such studies are in their infancy (see, e.g. Breitschwerdt et al 1993). With typical values of $\bar{B} \sim 1 \mu \mathrm{G}$ and $\rho \sim 10^{-27} \mathrm{~g} \mathrm{~cm}^{-3}$, a kinematic treatment will be valid for winds 
of speed in excess of about $100 \mathrm{~km} \mathrm{~s}^{-1}$. This outward advection of magnetic field may be partially offset near the disk by turbulent diamagnetism, which gives an effective velocity of field transport of a few kilometers per second towards the disk (if the diffusivity increases outwards), but for the larger wind velocities wind advection will dominate.

These problems were addressed in detail in the weak field approximation by Brandenburg et al (1993) and, with a rather different emphasis, by Elstner et al (1995). Brandenburg et al demonstrated that winds of plausible strength and geometry could drag out poloidal field lines almost radially into the halo and also move toroidal flux away from the disk. Moreover, by using realistic disk rotation curves for well-observed systems, and choosing appropriate (predominantly radial) wind velocity fields, solutions resembling the rather different halo fields of NGC 891 and NGC 4631, for example, can be generated without any careful "tuning." However, the halo field strengths are somewhat too low, and the field far from the disk makes too small an angle with the disk plane to provide a completely satisfactory model for NGC 4631.

However, a simple wind structure that is axisymmetric and varies smoothly with spherical polar angle $\theta$ may be inadequate; real galactic winds probably have considerably more structure, with streamers causing both azimuthal and latitudinal shear. Elstner et al (1995) presented a preliminary axisymmetric model (without azimuthal shear), with a wind velocity perpendicular to the disk and varying sinusoidally with distance from the rotation axis. They show that a short wavelength modulation $(1.5 \mathrm{kpc})$ can markedly affect the field geometry and that odd parity "dipolar" fields may even be stable for some parameter values. Further work with a more realistic model is needed to elucidate the relation between such calculations and real galactic flows.

A priori, a quasi-radial or $z$-wise shearing flow is unlikely to produce a halo field that is predominantly parallel to the disk plane, although such fields are observed in some "edge-on" galaxies (e.g. NGC 253). A problem concerning mechanisms that advect field from the disk is that the gas dragging it into the halo belongs to the rarefied, hot phase of the ISM, where the field strength is typically about $0.1 \mu \mathrm{G}$ (Kahn \& Brett 1993), and so additional amplification outside the disk is necessary. Shearing by localized outflows can only amplify the vertical component. Brandenburg et al (1995b) pointed out that galactic fountain flows, especially in active starburst galaxies, may have the correct topology (upflows connected in horizontal cross section and isolated downdrafts) for a topological pumping mechanism to produce a strong mean horizontal field high in the halo. With realistic parameters, they showed that this mechanism might produce horizontal fields at a height of several kpc above the disk that were of comparable strength to those in the disk. As yet, this mechanism has not been included in a 
global dynamo calculation. Magnetic buoyancy in the disk may also play a role in moving field into the halo, but this mechanism has not yet been adequately quantified.

In general, an outflow that is symmetric both azimuthally and with respect to the disk plane will preserve in the halo any global parity or symmetry properties of the disk field. Clearly, if the outflow lacks such symmetries (as seems quite possible, a priori), then this connection between disk and advected halo fields will be lost.

\section{MAGNETIC FIELD MODELS}

Only the dynamo theory for galactic magnetic fields has been developed sufficiently to provide models of magnetic fields in particular galaxies that can be confronted with observations. Therefore, our discussion below is inevitably more detailed in the case of the dynamo theory. Wherever possible, we also mention inferences from the primordial field theory, ignoring the conceptual difficulties discussed in Section 5.

\subsection{The Parity}

It is generally believed that galactic magnetic fields have an even parity. As discussed in Section 3.8.1, the field parity near the Sun most plausibly is even. There is some evidence for an even symmetry of the regular magnetic field in the edge-on galaxy NGC 253 (Beck et al 1994b). In mildly inclined galaxies, Faraday rotation measures for even and odd fields of equal strengths would differ only by a factor of 2 (Krause et al 1989a), which makes it difficult to distinguish between the two configurations. All conventional dynamo models indicate that the quadrupole parity must be dominant in galactic disks.

A uniform primordial magnetic field trapped by a protogalaxy, with arbitrary inclination to the rotation axis, produces an $\mathrm{S} 1$ component from the action of the radial gradient of the angular velocity $\Omega$ on $\bar{B}_{r}$ (which is then even in $z$ and nonaxisymmetric) and an A0 field from the action of $\partial \Omega / \partial z$ on $\bar{B}_{z}$ (which is odd and axisymmetric). Since $|\partial \Omega / \partial r| \gg|\partial \Omega / \partial z|$, at least during late stages of galactic evolution, the $\mathrm{S} 1$ field will become tightly wound and quickly decay because of reconnection. The resulting symmetry of a fossil field is then A0 or, possibly, a superposition of $\mathrm{S} 1$ and $\mathrm{A} 0$ configurations.

\subsection{Large-Scale Azimuthal Patterns}

Even the simplest asymptotic kinematic models of the mean-field dynamo in a thin disk have the promising property that only $m=0$ modes are excited in those galaxies where the field is observed to be axisymmetric (M31 and IC 342), whereas the $m=1$ mode is also excited (if it is not the fastest growing) in the 
galaxies with a dominant bisymmetric or mixed magnetic structure (e.g. M33, M51, and M81) (see Krasheninnikova et al 1990 and Ruzmaikin et al 1988a, $\mathrm{b}$ for reviews). The thinner the disk, the more readily the $m=1$ mode can be maintained (Ruzmaikin et al 1988a Section VII.8; Moss \& Brandenburg 1992). Weaker differential rotation is favorable for bisymmetric field generation. Even higher azimuthal modes might survive in galactic disks, e.g. the $m=2$ mode (Starchenko \& Shukurov 1989, Vallée 1992), which has a four-armed spiral pattern. An admixture of the $m=2$ mode may arise as a distortion of an $m=0$ field by a two-armed spiral pattern. An $m=2$ mode superimposed on a $m=0$ mode of similar amplitude would produce a pattern of the type possibly observed in NGC 6946 (Section 3.4).

The dominance of a bisymmetric field requires additional physical mechanisms to be invoked, as discussed in Sections 6.2 and 6.3; it seems, however, that these mechanisms are efficient only under certain conditions that can occur only in rare cases. Therefore, a general prediction of the galactic dynamo theory is that normally either axisymmetric magnetic structures (in the galaxies where only the $m=0$ mode is excited) or a superposition of $m=0$ and $m=1$ modes (where both are maintained) should be found. The former situation is encountered in M31 and IC 342, whereas the latter is represented by M51. An admixture of even higher $m$-modes cannot be excluded, as possibly seen in NGC 6946. Only in those galaxies that provide a suitable environment for a fine tuning of the dynamo (Sections 6.2 and 6.3) should a dominant bisymmetric field be expected, as exemplified by M81. An important factor in maintaining BSS seems to be tidal interaction with a companion galaxy (Section 6.2).

In general, this picture is reasonably consistent with observations that most galactic fields do not have simple structures. Note that a superposition of even two or three azimuthal modes may give an appearance of a rather irregular largescale magnetic field. So far, observations of only a few galaxies have been interpreted with allowance for such superpositions. We expect that new observations and analyses will extend the list of galaxies hosting mixed spiral structure.

A primordial magnetic field twisted by differential rotation is strongly dominated by the S1 or A0 modes (Section 3). An S0 field can arise only if it is assumed that the magnetic field had a very strong inhomogeneity across the protogalaxy (Sofue et al 1986), which appears to be a rather artificial requirement.

\subsection{Spiral Field Lines and Pitch Angles}

Plane-parallel magnetic fields with a dominant azimuthal component $\bar{B}_{\phi}$ prevail in spiral galaxies (see Section 3). This can be easily understood because differential rotation is strong in spiral galaxies (whether or not dynamos operate).

Dynamo theory predicts (Baryshnikova et al 1987), and observations of external galaxies show (Section 3.3), that the regular magnetic field must have 
the shape of a spiral, whether or not it is axisymmetric. Unlike spiral magnetic fields, a circular field produced within the galaxy (i.e. not supported by external currents) can not be maintained by any velocity field against turbulent magnetic diffusion. On average, the field must be a trailing spiral because differential rotation is important in producing $\bar{B}_{\phi}$ from $\bar{B}_{r}$. Of course, this does not preclude local deviations from a trailing spiral pattern, as observed, e.g. in M51 (Figure 1).

The pitch angle of the magnetic field $p$ is a readily observable parameter sensitive to details of the mechanism of magnetic field generation. Hence the magnetic pitch angle is an important diagnostic tool for theories of galactic magnetic fields. Magnetic pitch angles in spiral galaxies are observed to lie in the range $p=-\left(10^{\circ}-35^{\circ}\right)$ (Figure 8$)$. Galactic dynamo models even without spiral arms predict that $p$ is close to these values (Krasheninnikova et al 1989, Donner \& Brandenburg 1990, Elstner et al 1992, Panesar \& Nelson 1992). A simple estimate for a kinematic dynamo in a thin axisymmetric disk gives (Krasheninnikova et al 1989)

$$
p=\arctan \left(\bar{B}_{r} / \bar{B}_{\phi}\right) \simeq-\left(R_{\alpha} / R_{\omega}\right)^{1 / 2},
$$

and $p \simeq-20^{\circ}$ under typical conditions. Note that $\bar{B}_{r}$ and $\bar{B}_{\phi}$ have opposite signs because of the action of differential rotation, and so $p$ is negative (a trailing spiral). Asymptotic kinematic dynamo models using observed rotation curves have been applied to particular galaxies (see Ruzmaikin et al 1988a); the results agree fairly well with observations. Schultz et al (1994) discuss the dependence of the pitch angle on other parameters of turbulence.

It follows from Equations (3), (6), and (11) that $p \simeq-l / h$ (with $l$ as the turbulent scale). The pitch angle $|p|$ thus decreases with $r$ when $l=$ const, and $h$ increases with $r$. This behavior is also typical of dynamos in a flat disk (Elstner et al 1992, Panesar \& Nelson 1992) and is observed in spiral galaxies, as shown in Figure 8. The only exceptions are M81 and possibly also M33, both of which are candidates for bisymmetric magnetic structures due to interaction with companion galaxies (see Section 8.2).

As discussed in Section 3, magnetic pitch angles in spiral galaxies are surprisingly close to those of optical spiral arms, $p_{\text {SA }}$. Taken literally, Equation (11) implies that the equality $p \approx p_{\mathrm{SA}}$ is a mere quantitative coincidence because the two depend on different physical parameters. Numerical simulations of the $\alpha \omega$-dynamo with spiral shock waves (Panesar \& Nelson 1992) show that $p$ is quite insensitive to the presence of the shocks. The interplay between the magnetic and spiral patterns is far from being completely understood (Section 8.4) and, possibly, there are deeper physical reasons for the observed correspondence of the pitch angles.

Concerning the primordial field theory, a straightforward idea is that the pitch angle of a magnetic field frozen into a differentially rotating disk is a decreasing 
function of time and, after $N$ revolutions (with $N \simeq 30$ for the Solar vicinity in the Milky Way), we have $p \simeq-N^{-1} \operatorname{rad} \approx-2^{\circ}$, so that $|p| \ll\left|p_{\mathrm{SA}}\right|$. Furthermore, $|p|$ grows with $r$ insofar as angular velocity decreases with $r-\mathrm{a}$ trend opposite to that observed.

We note that the ASS fields observed in the spiral galaxies M31 and IC 342 and the magnetic spiral arms in NGC 6946 are directed inwards. For the edge-on galaxy NGC 253, a similar conclusion follows if one assumes that the magnetic field is also aligned with the spiral arms. As the direction of a dynamo-generated field is determined by that of the initial field, this dominance, if it were to be confirmed by better statistics, might clarify the nature of the seed field. For example, it could indicate the importance of battery effects (relying on galactic rotation). Within the framework of the primordial field theory, such a dominance would imply a hardly plausible correlation between the directions of the intergalactic field and the sense of galactic rotation.

\subsection{Spiral Arms and Magnetic Fields}

A standard understanding of the interaction between spiral arms and large-scale magnetic fields is largely based on the idea that the spiral shock compresses the magnetic field and aligns it with the spiral arm (Roberts \& Yuan 1970). This leads to a clear prediction that the regular magnetic field must be stronger at the inner edges of the arms and that there $p$ is closer to $p_{\mathrm{SA}}$ than in the interarm space. This picture was believed to be supported by the observation that the

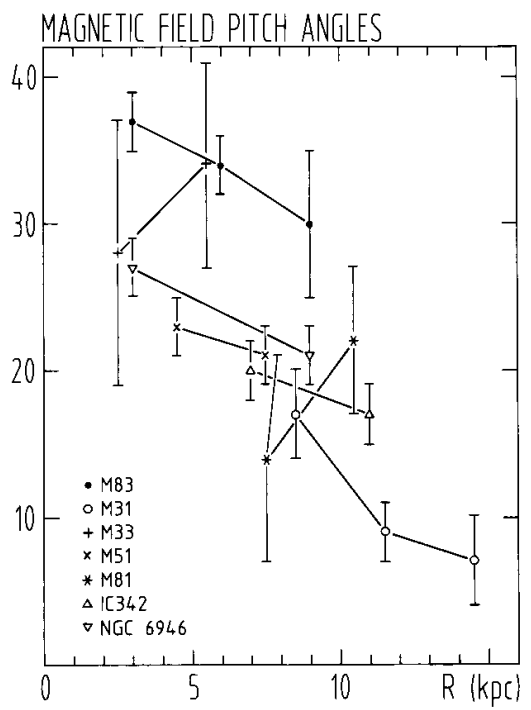

Figure 8 Observed radial variation of the magnetic pitch angle in the galaxy's plane averaged over azimuthal angle for several nearby spiral galaxies. (From Beck 1993.) 
regular magnetic field in the Milky Way is enhanced within the local arm and that magnetic fields observed in nearby galaxies are well aligned with the spiral arms. It is, however, noteworthy that $p \neq p_{\mathrm{SA}}$ near the Sun (Section 3.8), whereas the general alignment $p \approx p_{\mathrm{SA}}$ can arise from dynamo action without any shock compression (Section 8.3).

However, recent observations of most nearby galaxies indicate that the regular magnetic fields are observed to be stronger between the arms, whereas the total field strength is stronger in the arms (Sections 3.3 and 3.4). The implication is straightforward: The action of the spiral pattern on galactic magnetic fields is not as direct and simple as passive compression (at least in these galaxies). (We note also that it is difficult to understand how the primordial theory, which gives only a passive role to the magnetic field, can explain its enhancement between the arms. Possibly, streaming motions induced by spiral arms could help, but this possibility has not been studied.)

The compression of magnetic field in spiral arms becomes much weaker if a large fraction of the interstellar medium is filled with hot gas, which prevents large-scale shocks from occurring. Star formation in spiral arms must then be triggered by, e.g. more frequent collisions of gas clouds (Roberts \& Hausman 1984). The nearby spiral galaxies M51 and M81 exhibit strong density waves. In M51 prominent dust lanes, enhanced CO (García-Burillo et al 1993), and radio continuum emission at the inner edges of the optical spiral arms are indicators of narrow compression regions. In M81, however, the compression regions are much broader (Kaufman et al 1989) and can best be explained by the "cloudy" density-wave model of Roberts \& Hausman (1984).

Beck (1991) has proposed a qualitative model to explain enhanced field tangling in the arms. He assumes that the field lines are trapped by gas clouds. As the clouds enter a spiral arm, they are decelerated, and their number density, collision rate, and turbulent velocity increase, which gives rise to field tangling and enhanced total field. However, the "magnetic arms" observed between the optical arms of NGC 6946 (Section 3.4) cannot be understood by this model and need a global mechanism such as the dynamo. How to include spiral arms adequately into the theory of galactic magnetic fields remains an important unresolved problem.

\subsection{Dynamo Models for Individual Galaxies}

The predictions of $\alpha^{2} \Omega$-dynamo models are roughly consistent with the largescale field structures observed in spiral galaxies. In this section we discuss briefly a few individual galaxies for which detailed dynamo models have been developed and/or new problems have arisen.

Kinematic dynamo models for the Milky Way (see Ruzmaikin et al 1988a Sections VII.7 and VII.9) indicate that the axisymmetric mode is dominant, even though the $m=1$ mode can also be maintained if the half-thickness of the 
ionized disk is within a narrow range (500-700 pc near the Sun, but these values are model dependent). In view of the uncertainty concerning the generation of bisymmetric fields in spiral galaxies, an ASS seems more likely but the presence of the BSS cannot be excluded; a superposition of the two modes (MSS) is also possible.

The presence of reversals (Section 3.8) is often considered as an indication of a bisymmetric global structure of magnetic field in the Milky Way. We again stress that this is not true. The possibility of such reversals in an axisymmetric spiral field was demonstrated in a dynamo model for the Milky Way by Poezd et al (1993). Even this simplified model exhibits a reasonable agreement with observations, yielding two or three reversals whose positions along the radius roughly agree with those observed. According to Poezd et al, the reversals represent transient nonlinear magnetic structures (cf Section 6.4).

Both dynamo theory and observations agree that the large-scale magnetic field in M31 is axisymmetric. A notable feature of this galaxy is that both the gas and the large-scale magnetic field are concentrated within a narrow ring of about $10 \mathrm{kpc}$ radius (Section 3.2). The explanation provided by the dynamo models reviewed by Ruzmaikin et al (1988a, Section VII.7) relies on the rotation curve having a pronounced double-peaked shape (Deharveng \& Pellet 1975). However, recent interpretations (with better allowance for radial motions) have resulted in a much less pronounced minimum in the rotation curve (Kent 1989, Braun 1991). Even though the new rotation curve has not yet been incorporated into dynamo models, it can be guessed that the kinematic dynamo modes will no longer show any concentration into a ring. Thus, the ring-like structure of magnetic field in M31 probably arises during the nonlinear stage of the dynamo and is associated with a similar distribution of the interstellar gas (Dame et al 1993).

M81 is the only nearby galaxy for which a dominant bisymmetric magnetic field is firmly indicated by observations (Section 3). Apart from kinematic asymptotic dynamo models (Krasheninnikova et al 1989, Starchenko \& Shukurov 1989), a three-dimensional, nonlinear dynamo model has been developed for M81 based on the velocity field inferred from simulations of the interaction of this galaxy with its companion NGC 3077 (Moss et al 1993a). The interaction has been shown to result in a persistent bisymmetric structure. To reach a final conclusion about the nature of the magnetic field in M81, these numerical simulations must be extended to include better spatial resolution and a fully time-dependent representation of the velocity field. There is no minimum of polarized intensity observed near the probable location of the magnetic neutral line in M81 (Figure 2). Its absence probably indicates that the reversal in the BSS structure is rather abrupt, reminiscent of a contrast structure (see Section 6.2). 


\section{LAST WORDS}

We have attempted to draw together various strands contributing to our current understanding of galactic magnetism. We feel that neither dynamo nor fossil theory is at present in a satisfactory state. Nonetheless, we believe that, while the problems with the primordial theory are quite fundamental, ways of resolving the difficulties of the dynamo theory exist, in principle at least. A primordial field may nevertheless be important; for example, it can provide a seed field for a dynamo (see Section 5).

We note the following. Axisymmetric spiral structures and more complicated field structures arise naturally from dynamo models. Pitch angles lie in the correct range. Dynamo models give generically plausible large-scale spatial field structures, which are in some cases quite realistic, and which readily allow the detailed modeling of specific galaxies. Finally, on general grounds, field strengths of order the equipartition value, as observed, seem explicable. These points support our view that a coherent explanation of galactic magnetism will only be achieved via the further development of some form of dynamo theory.

It is now possible to include realistic models of the ISM, including detailed data on the spatial distributions of turbulent velocity and scale, the vertical gradient in the overall galactic rotation, galactic fountains, etc, in dynamo models; however, this remains to be done. A detailed comparison of theory with observations is becoming increasingly, both because the theory is beginning to give results that are sufficiently generic, reliable, and detailed, and because observations have reached the stage where they can seriously constrain many aspects of the theory. Reliable and high-resolution information about the complex magnetic structures found in the disks and halos of spiral galaxies is needed, together with an improved theory of depolarization mechanisms.

\section{ACKNOWLEDGMENTS}

The authors acknowledge the hospitality of the Observatory of Helsinki University, where the work was initiated, and of Nordita (Copenhagen), where it was finished. We are grateful to EM Berkhuijsen, who provided the data used in Figure 5 prior to publication. Partial financial support from the NATO grants CRG921273 and CRG1530959 is acknowledged. AS thanks the Mathematics Department of Manchester University and the Max-Planck-Institut für Radioastronomie for their hospitality during his work on the paper. AS and DS acknowledge partial financial support from grants 93-02-3638 and 95-0203724 from the Russian Foundation for Basic Research and MNP000/300 from the International Science Foundation.

Any Annual Review chapter, as well as any article cited in an Annual Review chapter, may be purchased from the Annual Reviews Preprints and Reprints service. 1-800-347-8007; 415-259-5017; email: arpr@class.org 


\section{Literature Cited}

Agafonov GI, Ruzmaikin AA, Sokoloff DD. 1988. Astron. Zh. 65: 523-28

Allen RJ, Sukumar S. 1990. In The Interstellar Medium in External Galaxies. Contributed Papers, ed. DJ Hollenbach, HA Thronson, pp. 263-67. NASA Conf. Publ. 3084

Andreassian RR. 1980. Astrofizika 16:707-13

Andreassian RR. 1982. Astrofizika 18:255-62

Armstrong JW, Rickett BJ, Spangler SR. 1995. Ap. J. 443:209-21

Baierlein R. 1978. MNRAS 184:843-70

Balbus SA, Hawley JF. 1992. Ap. J. 392:662-66

Baryshnikova Y, Ruzmaikin A, Sokoloff DD, Shukurov A. 1987. Astron. Astrophys. 177:27-41

Beck R. 1982. Astron. Astrophys. 106:121-32

Beck R. 1991. Astron. Astrophys. 251:15-26

Beck R. 1993. In Cosmic Dynamo, ed. F Krause, K-H Rädler, G Rüdiger, pp. 283-97. Dordrecht: Kluwer

Beck R, Carilli CL, Holdaway MA, Klein U. 1994b. Astron. Astrophys. 292:409-24

Beck R, Hoernes P. 1996. Nature 379:47-49

Beck R, Klein U, Wielebinski R. 1987. Astron. Astrophys. 186:95-98

Beck R, Loiseau N, Hummel E, Berkhuisen EM, Gräve R, Wielebinski R. 1989. Astron. Astrophys. 222:58-68

Beck R, Poezd AD, Shukurov A, Sokoloff DD. 1994a. Astron. Astrophys. 289:94-100

Belyanin M, Sokoloff DD, Shukurov A. 1993. Geophys. Astrophys. Fluid Dyn. 68:237-61

Belyanin MP, Sokoloff DD, Shukurov AM. 1994. Russ. J. Math. Phys. 2:149-74

Berkhuijsen EM, Bajaja E, Beck R. 1993. Astron. Astrophys. 279:359-75

Berkhuijsen EM, Golla G, Beck R. 1991. In The Interstellar Disk-Halo Connection in Galaxies. Proc. IAU Symp. 144, ed. H Bloemen, pp. 233-36. Dordrecht: Kluwer

Beuermann K, Kanbach G, Berkhuijsen EM. 1985. Astron. Astrophys. 153:17-34

Biermann L. 1950. Z. Naturforsch. 5a:65-71

Binney J, Tremaine S. 1987. Galactic Dynamics. Princeton: Princeton Univ. Press

Bloemen JB, Strong AW, Blitz L, Cohen RS, Dame TM, et al. 1986. Astron. Astrophys. 154:25-41

Boulares A, Cox DP. 1990. Ap. J. 365:544-58

Bowyer S, Lieu R, Sidher SD, Lampton M, Knude J. 1995. Nature 375:212-14

Brandenburg A, Donner KJ, Moss D, Shukurov A, Sokoloff DD, Tuominen I. 1992. Astron. Astrophys. 259:453-61

Brandenburg A, Donner KJ, Moss D, Shukurov A, Sokoloff DD, Tuominen I. 1993. Astron. Astrophys. 271:36-50
Brandenburg A, Moss D, Shukurov A. 1995 b. MNRAS 276:651-62

Brandenburg A, Nordlund $\AA$, Stein RF, Torkelsson U. 1995a. Ap. J. 446:741-54

Brandenburg A, Tuominen I, Krause F. 1990. Geophys. Astrophys. Fluid Dyn. 50:95-112

Brandenburg A, Zweibel EG. 1995. Ap. J. 448:734-41

Braun R. 1991. Ap. J. 372:54-66

Breitschwerdt D, Völk HJ, Ptuskin V, Zirakashvili V. 1993. In The Cosmic Dynamo, ed. F Krause, K-H Rädler, G Rüdiger, pp. 415-19. Dordrecht: Kluwer

Briggs FH, Wolfe AM, Liszt HS, Davis MM, Turner KL. 1989. Ap. J. 341:650-57

Buczilowski UR, Beck R. 1991. Astron. Astrophys. 241:47-56

Burn BJ. 1966. MNRAS 133:67-83

Camenzind M, Lesch H. 1994. Astron. Astrophys. 284:411-23

Carilli CL, Holdaway MA, Ho PTP, DePree CG. 1992. Ap. J. Lett. 399:L59-62

Carvalho JC. 1992. Astron. Astrophys. 261:34852

Cattaneo F, Vainshtein SI. 1991. Ap. J. Lett. 376:L21-24

Chakrabarti SK, Rosner R, Vainshtein SI. 1994. Nature 368:434-36

Cheng B, Schramm DN, Truran JW. 1994. Phys. Rev. D 49:5006-18

Chi X, Wolfendale AW. 1993. Nature 362:61011

Chiba M, Lesch H. 1994. Astron. Astrophys. 284:731-48

Chiba M, Tosa M. 1990. MNRAS 244:714-26

Clegg AW, Cordes JM, Simonetti JH, Kulkarni SR. 1992. Ap. J. 386:143-57

Dahlem M, Aalto S, Klein U, Booth R, Mebold U, Wielebinski R, Lesch H. 1990. Astron. Astrophys. 240:237-46

Dahlem M, Lisenfeld U, Golla G. 1995. Ap. J. 444:119-28

Dame TM, Koper E, Israel FP, Thaddeus P. 1993. Ap. J. 418:730-42

Davidson JA, Schleuning D, Dotson JL, Dowell CD, Hildebrand RH. 1995. In Airborne Astron. Symp. on the Galactic Ecosystem, ed. MR Haas, JA Davidson, EF Erickson, pp. 225-34. San Francisco: Astron. Soc. Pac. Conf. Ser. 73

Deharveng JM, Pellet A. 1975. Astron. Astrophys. 38:15-28

Dettmar R-J. 1990. Astron. Astrophys. 232:L15-18

Dickey JM, Brinks E, Puche D. 1992. Ap. J. 385:501-11

Dobler W, Poezd A, Shukurov A. 1996. Astron. 
Astrophys. In press

Donner KJ, Brandenburg A. 1990. Astron. Astrophys. 240:289-97

Dumke M, Krause M, Wielebinski R, Klein U. 1995. Astron. Astrophys. 302:691-703

Ehle M. 1995. Eigenschaften des diffusen interstelleren Mediums in den nahen Galaxien M51, M83, und NGC 1566. PhD Thesis. Univ. Bonn

Ehle M, Beck R. 1993. Astron. Astrophys. 273:45-64

Ehle M, Beck R, Haynes RF, Vogler A, Pietsch W, Elmouttie M, Ryder S. 1996. Astron. Astrophys. 306:73-85

Elmouttie M, Haynes RF, Jones KL, Ehle M, Beck R, Wielebinski R. 1995. MNRAS 275:L53-59

Elstner D, Golla G, Rüdiger G, Wielebinski R. 1995. Astron. Astrophys. 297:77-82

Elstner D, Meinel R, Beck R. 1992. Astron. Astrophys. Suppl. 94:587-600

Elstner D, Rüdiger G, Schultz M. 1996. Astron. Astrophys. 306:740-46

Enqvist K, Rez AI, Semikoz V. 1995. Nucl. Phys. B 436:49-64

Enqvist K, Semikoz V, Shukurov A, Sokoloff D. 1993. Phys. Rev. D 48:4557-61

Falgarone E, Lis DC, Phillips TG, Pouquet A, Porter DH, Woodward PR. 1994. Ap. J. 436:728-40

Ferrière K. 1993. Ap. J. 404:162-84

Field GB. 1996. In The Physics of the Interstellar Medium and Intergalactic Medium, ed. A Ferrara et al, pp. 1-2. Astron. Soc. Pac. Conf. Ser. Vol. 80

Fitt AJ, Alexander P. 1993. MNRAS 261:445-52

Frisch U, Pouquet A, Léorat J, Mazure A. 1975. J. Fluid Mech. 68:769-78

Garcia-Burillo S, Guélin M, Cernicharo J. 1993. Astron. Astrophys. 274:123-47

Gardner FF, Morris D, Whiteoak JB. 1969. Aust. J. Phys. 22:813-19

Gavazzi G, Boselli A, Kennicutt R. 1991. Astron. J. 101:1207-30

Golla G, Beck R. 1990. In The Interstellar DiskHalo Connection in Galaxies. Poster Proc. IAU 144, ed. H Bloemen, pp. 47-48. Leiden Obs.

Golla G, Hummel E. 1994. Astron. Astrophys. 284:777-92

Grasso D, Rubinstein HR. 1995. Astroparticle Phys. 3:95-102

Gräve R, Beck R. 1988. Astron. Astrophys. 192:66-76

Gräve R, Klein U, Wielebinski R. 1990. Astron. Astrophys. 238:39-49

Gruzinov AV, Diamond PH. 1994. Phys. Rev. Lett. 72:1651-53

Han JL, Qiao GJ. 1994. Astron. Astrophys. 238:759-72
Hanasz M, Chiba M. 1993. MNRAS 266:545-68

Hanasz M, Lesch H. 1993. Astron. Astrophys. 278:561-68

Hanasz M, Lesch H, Krause M. 1991. Astron. Astrophys. 243:381-85

Harnett JI, Haynes RF, Klein U, Wielebinski R. 1989. Astron. Astrophys. 216:39-43

Harnett JI, Haynes RF, Wielebinski R, Klein U. 1990. Proc. Astron. Soc. Aust. 8:257-60

Harrison ER. 1970. MNRAS 147:279-86

Haynes RF, Klein U, Wielebinski R, Murray JD. 1986. Astron. Astrophys. 159:22-32

Heiles C. 1976. Annu. Rev. Astron. Astrophys. 14:1-22

Heiles C. 1989. Ap. J. 336:808-21

Heiles C. 1996. In Polarimetry of the Interstellar Medium, ed. W Roberge, D Whittet. San Francisco: Astron. Soc. Pac. In press

Heiles C, Goodman AA, McKee CF, Zweibel EG. 1993. In Protostars and Planets III, ed. EH Levy, JI Lunine, pp. 279-326. Tuscon: Univ. Ariz. Press

Higdon JC. 1984. Ap. J. 285:109-23

Hildebrand RH, Davidson JA. 1994. In The $\mathrm{Nu}$ clei of Normal Galaxies, ed. R Genzel, AI Harris, pp. 199-203. Dordrecht: Kluwer

Hildebrand RH, Gonatas DP, Platt SR, Wu XD, Davidson JA, et al. 1990. Ap. J. 362:114-19

Horellou C, Beck R, Berkhuijsen EM, Krause M, Klein U. 1992. Astron. Astrophys. 265:417-28

Horellou C, Beck R, Klein U. 1990. In Galactic and Intergalactic Magnetic Fields, ed. R Beck, PP Kronberg, R Wielebinski, pp. 21112. Dordrecht: Kluwer

Hough JH. 1996. In Polarimetry of the Interstellar Medium, ed. W Roberge, D Whittet. San Francisco: Astron. Soc. Pac. In press

Hoyle F. 1969. Nature 223:936

Hummel E. 1986. Astron. Astrophys. 160:L4 L6

Hummel E. 1990. In Windows on Galaxies, ed. G Fabbiano, JS Gallagher, A Renzini, pp. 141-55. Dordrecht: Kluwer

Hummel E, Beck R. 1995. Astron. Astrophys. 303:691-704

Hummel E, Beck R, Dahlem M. 1991a. Astron. Astrophys. 248:23-29

Hummel E, Beck R, Dettmar R-J. 1991b. Astron. Astrophys. Suppl. 87:309-17

Hummel E, Davies RD, Wolstencroft RD, van der Hulst JM, Pedlar A. 1988b. Astron. Astrophys. 199:91-104

Hummel E, Krause M, Lesch H. 1989. Astron. Astrophys. 211:266-74

Hummel E, Lesch H, Wielebinski R, Schlickeiser R. 1988a. Astron. Astrophys. 197:L2931

Kahn FD, Brett L. 1993. MNRAS 263:37-48

Kaisig M, Rüdiger G, Yorke HW. 1993. Astron. 
Astrophys. 274:757-64

Kaufman M, Bash FN, Hine B, Rots AH, Elmegreen DM, Hodge PW. 1989. Ap. J. 345:674-96

Kazantsev AP. 1968. Sov. Phys. JETP 26:103134

Kent SM. 1989. Astron. J. 97:1614-21

Kitchatinov LL, Pipin VV, Rüdiger G. 1994. Astron. Nachr. 315:157-70

Kitchatinov LL, Rüdiger G. 1992. Astron. Astrophys. 260:494-98

Klein U, Haynes RF, Wielebinski R, Meinert D. 1993. Astron. Astrophys. 271:402-12

Klein U, Wielebinski R, Morsi HW. 1988. Astron. Astrophys. 190:41-46

Ko CM, Parker EN. 1989. Ap. J. 341:828-31

Kraichnan RH. 1965. Phys. Fluids 8:1385-86

Krasheninnikova Y, Ruzmaikin A, Sokoloff D, Shukurov A. 1989. Astron. Astrophys. 213:19-28

Krasheninnikova Y, Ruzmaikin A, Sokoloff D, Shukurov A. 1990. Geophys. Astrophys. Fluid Dyn. 50:131-46

Krause F. 1967. Eine Lösung des Dynamoproblems auf der Grundlage einer linearen Theorie der magnetohydrodynamischen Turbulenz. Univ. Jena: Habilitationsschrift

Krause F, Rädler K-H. 1980. Mean-Field Electrodynamics and Dynamo Theory. Berlin: Akademie-Verlag; Oxford: Pergamon

Krause F, Rüdiger G. 1975. Sol. Phys. 42:10719

Krause M. 1990. In Galactic and Intergalactic Magnetic Fields, ed. R Beck, PP Kronberg, R Wielebinski, pp. 187-96. Dordrecht: Kluwer

Krause M. 1993. In The Cosmic Dynamo, ed. F Krause, K-H Rädler, G Rüdiger, pp. 305-10. Dordrecht: Kluwer

Krause M, Beck R, Hummel E. 1989b. Astron. Astrophys. 217:17-30

Krause M, Hummel E, Beck R. 1989a. Astron. Astrophys. 217:4-16

Kronberg PP. 1994. Rep. Prog. Phys. 57:325-82

Kronberg PP, Perry JJ. 1982. Ap. J. 263:518-32

Kronberg PP, Perry JJ, Zukowski ELH. 1992. Ар. J. 387:528-35

Kulsrud R. 1986. In Plasma Astrophysics, ed. TD Guyenne, LM Zeleny, pp. 531-37. Paris: ESA Publ. SP-251

Kulsrud RM, Anderson SW. 1992. Ap. J. 396:606-30

Kuzanyan KM, Sokoloff D. 1993. Astrophys. Space Sci. 208:245-52

Landau LD, Lifshitz EM. 1969. Mechanics. Oxford: Pergamon. 2nd Ed.

LeBlanc VG, Kerr D, Wainwright J. 1995. Class. Quantum Grav. 12:513-41

Longair MS. 1994. High Energy Astrophysics, Cambridge: Cambridge Univ. Press, 2:29296
Lyne AG, Smith FG. 1989. MNRAS 237:533-41

McIvor I. 1977. MNRAS 178:85-100

McKee CF, Zweibel EG. 1995. Ap. J. 440:68696

McKee CF, Zweibel EG, Goodman AA, Heiles C. 1993. In Protostars and Planets III, ed. EH Levy, JI Lunine, pp. 327-67. Tucson: Univ. Ariz. Press

Meinel R, Elstner D, Rüdiger G. 1990. Astron. Astrophys. 236:L33-35

Meneguzzi M, Frisch U, Pouquet A. 1981. Phys. Rev. Lett. 47:1060-64

Mestel L. 1966. MNRAS 133:265-84

Mestel L. 1985. Physica Scripta T11:53-58

Mestel L. 1989. In Accretion Disks and Magnetic Fields in Astrophysics, ed. G Belvedere, pp. 151-63. Dordrecht: Kluwer

Mestel L, Roxburgh IW. 1962. Ap. J. 136:61526

Mestel L, Subramanian K. 1991. MNRAS 248:677-87

Mishustin IN, Ruzmaikin AA. 1971. Sov. Phys. JETP 61:441-44

Moffatt HK. 1972. J. Fluid Mech. 53:385-99

Moffatt HK. 1978. Magnetic Field Generation in Electrically Conducting Fluids. Cambridge: Cambridge Univ. Press

Moffatt HK. 1979. Geophys. Astrophys. Fluid Dyn. 14:147-66

Morris D, Berge GL. 1964. Ap. J. Lett. 139:1388-93

Moss D. 1995. MNRAS 275:191-94

Moss D. 1996. Astron. Astrophys. In press

Moss D, Brandenburg A. 1992. Astron. Astrophys. 256:371-74

Moss D, Brandenburg A, Donner KJ, Thomasson M. 1993a. Ap. J. 409:179-89

Moss D, Brandenburg A, Donner KJ, Thomasson M. 1993b. In The Cosmic Dynamo, ed. F Krause, K-H Rädler, G Rüdiger, pp. 339-43. Dordrecht: Kluwer

Moss D, Brandenburg A, Tuominen I. 1991. Astron. Astrophys. 247:576-79

Moss D, Shukurov AM. 1996. MNRAS 279:229-39

Moss D, Tuominen I. 1990. Geophys. Astrophys. Fluid Dyn. 50:113-20

Moss D, Tuominen I, Brandenburg A. 1990. Astron. Astrophys. 228:284-94

Neininger N. 1992. Astron. Astrophys. 263:3036

Neininger N, Beck R, Sukumar S, Allen RJ. 1993. Astron Astrophys. 274:687-98

Neininger N, Horellou C. 1996. In Polarimetry of the Interstellar Medium, ed. W Roberge, D Whittet. San Francisco: Astron. Soc. Pac. In Press

Neininger N, Klein U, Beck R, Wielebinski R. 1991. Nature 352:781-82

Nelson AH. 1988. MNRAS 233:115-21 
Niklas S, Klein U, Wielebinski R. 1995. Astron. Astrophys. 293:56-63

Nordlund A, Brandenburg A, Jennings RL, Rieutord M, Ruokolainen J, et al. 1992. Ap. J. 392:647-52

Nordlund A, Galsgaard K, Stein RF. 1994. In Solar Surface Magnetic Fields, ed. RJ Rutten, CJ Schrijver, pp. 471-98. Dordrecht: Kluwer

Nozakura T. 1993. MNRAS 260:861-74

Ohno H, Shibata S. 1993. MNRAS 262:953-62

Oren AL, Wolfe AM. 1995. Ap. J. 445:624-41

Otmianowska-Mazur K, Chiba M. 1995. Astron. Astrophys. 301:41-54

Otmianowska-Mazur K, Urbanik M. 1994. Geophys. Astrophys. Fluid Dyn. 75:61-75

Panesar JS, Nelson AH. 1992. Astron. Astrophys. 264:77-85

Parker EN. 1955. Ap. J. 122:293-314

Parker EN. 1971. Ap. J. 163:252-78

Parker EN. 1973. Astrophys. Space Sci. 22:279_ 91

Parker EN. 1979. Cosmical Magnetic Fields. Oxford: Clarendon

Parker EN. 1992. Ap. J. 401:137-45

Perry JJ. 1994. In Cosmical Magnetism. Contributed Papers in Honour of Prof. L. Mestel, ed. D Lynden-Bell, pp. 144-51. Cambridge: Inst. Astron.

Perry JJ, Watson AM, Kronberg PP. 1993. Ap. J. 406:407-19

Piddington JH. 1970. Aust. J. Phys. 23:731-50

Pietsch W. 1994. In Panchromatic View of Galaxies, ed. G Hensler, C Theis, JS Gallagher, pp. 137-154. Gif-sur-Yvette: Editions Frontières

Poezd A, Shukurov A, Sokoloff D. 1993. MNRAS 264:285-97

Pohl M. 1993. Astron. Astrophys. 279:L17-20

Pouquet A, Frisch U, Léorat J. 1976. J. Fluid Mech. 77:321-54

Pudritz RE, Silk J. 1989. Ap. J. 342:650-59

Quashnock JM, Loeb A, Sprengel DN. 1989. Ap. J. Lett. 344:L49-51

Rädler K-H. 1969. Geod. Geophys. Veröff. II 13:131-35

Rand RJ, Kulkarni SR. 1989. Ap. J. 343:760-72

Rand RJ, Kulkarni SR, Hester JJ. 1990. Ap. J. Lett. 352:L1-L4

Rand RJ, Lyne AG. 1994. MNRAS 268:497-505

Ratra B. 1992. Ap. J. 391:L1-L4

Reuter H-P, Klein U, Lesch H, Wielebinski R, Kronberg PP. 1994. Astron. Astrophys. 282:724-30; 293:287-88 (Erratum).

Reuter H-P, Krause M, Wielebinski R, Lesch H. 1991. Astron. Astrophys. 248:12-22

Roberge W, Whittet D. 1996. Polarimetry of the Interstellar Medium. San Francisco: Astron. Soc. Pac.

Roberts PH, Soward AM. 1975. Astron. Nachr. 296:49-64
Roberts PH, Soward AM. 1992. Аnnu. Rev. Fluid Dyn. 24:459-512

Roberts WW, Hausman MA. 1984. Ap. J. 277:744-67

Roberts WW, Yuan C. 1970. Ap. J. 161:877-902

Rüdiger G, Elstner D, Schultz M. 1993. Astron. Astrophys. 270:53-59

Rüdiger G, Kitchatinov LL. 1993. Astron. Astrophys. 269:581-88

Ruzmaikin AA, Shukurov AM. 1982. Astrophys. Space Sci. 82:397-407

Ruzmaikin AA, Shukurov AM, Sokoloff DD. 1988a. Magnetic Fields of Galaxies. Dordrecht: Kluwer

Ruzmaikin A, Shukurov AM, Sokoloff D. 1988b. Nature 336:341-47

Ruzmaikin AA, Sokoloff DD. 1977. Astron. Astrophys. 58:247-53

Ruzmaikin AA, Sokoloff DD. 1979. Astron. Astrophys. 78:1-6

Ruzmaikin AA, Sokoloff DD, Kovalenko AV. 1978. Sov. Astron. 22:395-401

Ruzmaikin A, Sokoloff D, Shukurov A. 1988c. J. Fluid Mech. 197:39-56

Ruzmaikin A, Sokoloff D, Shukurov A, Beck R. 1990. Astron. Astrophys. 230:284-92

Rybicki GB, Lightman AP. 1979. Radiative Processes in Astrophysics. New York: Wiley

Scarrott SM, Draper PW, Stockdale DP, Wolstencroft RD. 1993. MNRAS 249:L7-12

Scarrott SM, Rolph CD, Semple DP. 1990. In Galactic and Intergalatic Magnetic Fields, ed. R Beck, PP Kronberg, R Wielebinski, pp. 245-51. Dordrecht: Kluwer

Scarrott SM, Rolph CD, Walstencraft RD, Tadhunter CN. 1991. MNRAS 224:16-20

Scarrott SM, Ward-Thompson D, WarrenSmith RF. 1987. MNRAS 224:299-305

Schlegel EM. 1994. Ap. J. 434:523-35

Schmitt D, Rüdiger G. 1992. Astron. Astrophys. 264:319-26

Schoofs S, 1992. Hochaufgelöste VLABeobachtungen im Radiokontinuum zum Magnetfeld der Spiralgalaxie M81. Diploma Thesis, Univ. Bonn

Schultz M, Elstner D, Rüdiger G. 1994. Astron. Astrophys. 286:72-79

Sciama DW. 1994. In Cosmical Magnetism. Contributed Papers in Honour of Prof. LMestel, ed. D Lynden-Bell, pp. 128-33. Cambridge: Inst. Astron.

Segalovitz A, Shane WW, de Bruyn AG. 1976. Nature 264:222-26

Simard-Normandin M, Kronberg PP. 1979. Nature 279:115-18

Simard-Normandin M. Kronberg PP. 1980. Ap. J. 242:74-94

Sofue Y. 1987. Publ. Astron. Soc. Jpn. 39:54757

Sofue Y, Fujimoto M. 1993. Ap. J. 265:722-29 
Sofue Y, Fujimoto M, Wielebinski R. 1986. Annu. Rev. Astron. Astrophys. 24:459-97

Sofue Y, Klein U, Beck R, Wielebinski R. 1985. Astron. Astrophys. 144:257-60

Sofue Y, Takano T. 1981. Publ. Astron. Soc. Jpn. 33:47-55

Soida M, Urbanik U, Beck R. 1996. Astron. Astrophys. In press

Sokoloff D, Ruzmaikin A, Shukurov A. 1990. In Galactic and Intergalactic Magnetic Fields, ed. R Beck, PP Kronberg, R Wielebinski, pp. 499-503. Dordrecht: Kluwer

Sokoloff DD, Shukurov A. 1990. Nature 347:51-53

Sokoloff D, Shukurov A, Krause M. 1992. Astron. Astrophys. 264:396-405

Spencer SJ, Cram LG. 1992. Ap. J. 400:484501

Starchenko SV, Shukurov AM. 1989. Astron. Astrophys. 214:47-60

Steenbeck M, Krause F, Rädler K-H. 1966. Z. Naturforsch. 21a:369-76; see also transl. in PH Roberts, M Stix. 1971. The Turbulent Dynamo, Tech. Note 60. Boulder, Co: NCAR

Stepinski TF, Levy EH. 1988. Ap. J. 331:416-34

Subramanian K, Mestel L. 1993. MNRAS 265:649-54

Sukumar S, Allen RJ. 1989. Nature 340:537-39

Sukumar S, Allen RJ. 1991. Ap. J. 382:100-7

Tajima T, Cable S, Shibata K, Kulsrud R. 1992. Ap. J. 390:309-21

Tao L, Cattaneo F, Vainshtein SI. 1993. In Solar and Planetary Dynamos, ed. MRE Proctor, PC Matthews, AM Rucklidge, pp. 303-10. Cambridge: Cambridge Univ. Press

Thomasson M, Donner KJ. 1993. Astron. Astrophys. 272:153-60

Thomson RC, Nelson AH. 1980. MNRAS 191:863-70

Tinbergen J. 1996. Astronomical Polarimetry. Cambridge: Cambridge Univ. Press

Tosa M, Fujimoto M. 1978. Publ. Astron. Soc. Jpn. 30:315-25

Turner MS, Widrow LM. 1988. Phys. Rev. D 37:2743-54

Turnshek DA, Wolfe AM, Lanzetta KM, Briggs FH, Cohen RD, et al. 1989. Ap. J. 344:567-96

Urbanik M, Otmianowska-Mazur K, Beck R. 1994. Astron. Astrophys. 287:410-18

Vachaspati T. 1991. Phys. Lett. B 265:258-61

Vainshtein SI, Cattaneo F. 1992. Ap. J. 393:16571

Vainshtein SI, Ruzmaikin AA. 1971. Astron. Zh.
48:902-9 (Sov. Astron. 16:365-67)

Vallée JP. 1980. Astron. Astrophys. 86:251-53

Vallée JP. 1983. Astron. Astrophys. 124:147-50

Vallée JP. 1986. Astron. J. 91:541-45

Vallée JP. 1991. Ap. J. 366:450-54

Vallée JP. 1992. Astron. Astrophys. 255:100-4

Vallée JP. 1993. Ap. J. 419:670-73

Vallée JP. 1994. Ap. J. 433:778-79

Vallée JP. 1996. Astron. Astrophys. In press

Vallée JP, Kronberg PP. 1973. Nat. Phys. Sci. 246:49-51

Vallée JP, Kronberg PP. 1975. Astron. Astrophys. 43:233-42

Vallée JP, Simard-Normandin M, Bignell RC. 1988. Ap. J. 331:321-24

Vázquez-Semadeni E, Passot T, Pouquet A. 1995. Ap. J. 441:702-25

Verschuur GL. 1979. Fund. Cosmic Phys. 5:113-91

Walker MR, Barenghi CF. 1994. Geophys. Astrophys. Fluid Dyn. 76:265-81

Welter GL, Perry JJ, Kronberg PP. 1984. Ap. J. 279:19-39

White RL, Kinney AL, Becker RH. 1993. Ap. J. 407:456-69

Wielebinski R, Krause F. 1993. Astron. Astrophys. Rev. 4:449-85

Wolfe AM. 1988. In QSO Absorption Lines, ed. JC Blades, DA Turnshek, CA Norman, pp. 297-317. Cambridge: Cambridge Univ. Press

Wolfe AM, Lanzetta KM, Oren AL. 1992. Ap. J. 388:17-22

Wolfe AM, Turnshek DA, Lanzetta KM, Lu L. 1993. Ap. J. 404:480-510

Yoshizawa A, Yokoi N. 1993. Ap. J. 407:54048

Zeldovich YaB. 1957. Sov. Phys. JETP 4:46062

Zeldovich YaB. 1965. Sov. Phys. JETP 48:98688

Zeldovich YaB, Molchanov SA, Ruzmaikin AA, Sokoloff DD. 1988. Sov. Sci. Rev. C Math. Phys. 7:1-110

Zeldovich YaB, Novikov ID. 1982. The Structure and Evolution of the Universe. Chicago: Chicago Univ. Press

Zeldovich YaB, Ruzmaikin AA, Sokoloff DD. 1983. Magnetic Fields in Astrophysics. New York: Gordon \& Breach

Zweibel EG. 1988. Ap. J. Lett. 329:L1-L4

Zweibel EG, McKee CF. 1995. Ap. J. 439:77992 